\title{
A Hybrid Real/Ideal Gas Mixture Computational Framework to Capture Wave Propagation in Liquid Rocket Combustion Chamber Conditions
}

\author{
Simone $D^{\prime}$ Alessandro ${ }^{1, *}$, , Marco Pizzarelli ${ }^{2}$ and Francesco Nasuti ${ }^{1}[$ \\ 1 Department of Mechanical and Aerospace Engineering, Sapienza University of Rome, Via Eudossiana 18, \\ 00184 Rome, Italy; francesco.nasuti@uniroma1.it \\ 2 Italian Space Agency (ASI), Via del Politecnico snc, 00133 Rome, Italy; marco.pizzarelli@asi.it \\ * Correspondence: simone.dalessandro@uniroma1.it
}

Citation: D'Alessandro, S.; Pizzarelli, M.; Nasuti, F. A Hybrid Real/Ideal Gas Mixture Computational Framework to Capture Wave Propagation in Liquid Rocket Combustion Chamber Conditions. Aerospace 2021, 8, 250. https:// doi.org/10.3390/aerospace8090250

Academic Editor: Francesco Battista

Received: 19 July 2021

Accepted: 27 August 2021

Published: 4 September 2021

Publisher's Note: MDPI stays neutral with regard to jurisdictional claims in published maps and institutional affiliations.

Copyright: (c) 2021 by the authors. Licensee MDPI, Basel, Switzerland. This article is an open access article distributed under the terms and conditions of the Creative Commons Attribution (CC BY) license (https:// creativecommons.org/licenses/by/ $4.0 /)$.

\begin{abstract}
The present work focuses on the development of new mathematical and numerical tools to deal with wave propagation problems in a realistic liquid rocket chamber environment. A simplified real fluid equation of state is here derived, starting from the literature. An approximate Riemann solver is then specifically derived for the selected conservation laws and primitive variables. Both the new equation of state and the new Riemann solver are embedded into an in-house one-dimensional CFD solver. The verification and validation of the new code against wave propagation problems are then performed, showing good behavior. Although such problems might be of interest for different applications, the present study is specifically oriented to the low order modeling of high-frequency combustion instability in liquid-propellant rocket engines.
\end{abstract}

Keywords: low-order modeling; real fluid; wave propagation; equation of state; Riemann solver; liquid rocket engines; combustion instability; thermo-acoustics

\section{Introduction}

Recent studies have provided evidence on how the injector elements of liquid rocket engines play a central role in the thermo-, hydro-, and gas-dynamic coupling that takes place during high frequency combustion instability [1-3]. In particular, the commonly used shear-coaxial injector shows an attitude to cyclically accumulate and release fresh fuel pockets. Such a behavior is most likely caused by the intrinsic nature of the injectors themselves, which work with a radial density gradient in the recess area, i.e., the zone where fuel and oxidizers mix with each other. When fluctuations occur, axial pressure gradients develop inside the injectors because of their basically one-dimensional shape, and the baroclinic torque arising from the coupling of the two gradients generates vortexes in turn in the recess. Since the intensity of the pressure waves during thermo-acoustic oscillations can be strong, pressure waves coming from the chamber and traveling upstream in the injector can significantly slow the flow down. In such a case, vortexes may form, trapping pockets of fresh propellant, which struggle to flow downstream, causing an accumulation process to take place at the recess location. Afterwards, when the pressure waves return, traveling downstream, the accumulated pockets are pushed into the hot gases in the chamber where they burn, releasing heat and generating new pressure waves in turn. So, the process becomes self-sustained.

This work is mainly focused on acoustic phenomena and their correct modeling. In this sense, attention has to be paid to two major sub-models, namely, the real fluid mixture equation of state (EoS) and the proper numerical approach. For both of them, several different approaches are available in the literature, spanning from very simple to very complicated models, depending on the problem that is being dealt with.

Concerning the equation of state, the most simple way to model a real fluid mixture is to assume one cubic EoS for each species, mixing them afterwards using an ideal gas mixing 
rule. In particular, the use of Amagat's mixing rule [4] seems quite promising [5]. A further complication is to assume a cubic model cast for the entire mixture. In such a way, mixing is performed by means of species interaction indexes within the EoS [6-8]. Such a kind of modeling drops completely any kind of idealization and is quite widespread in the acoustics community, [9-13]. The last step of complication consists of modeling the Helmholtz free energy. Theoretically, such an elegant formulation is the most accurate, yet also the most complicated and computationally intensive among all the others. Furthermore, it is quite recent as a formulation, so many of the needed species interaction coefficients employed in it are not yet available in the literature [14,15].

For the mentioned reasons, a cubic EoS for the entire real gas mixture is chosen as a starting point [16]; then, it is significantly simplified, as described in the following sections.

To solve the conservation laws, a finite volumes numerical approach based on an approximated Riemann solver (RS) is selected. As for the EoS, a large number of Riemann solvers is available in the literature, depending on the problem under investigation. Most of them are reported in detail in [17]. In the literature, a popular approximated RS is the Roe solver, whose original formulation $[18,19]$ was already extended to generalized EoS by Glaister [20]. Such a solver is re-derived in the following sections in different primitive variables, with respect to the original formulation, for consistency with the primitive variables that are considered in the formulation presented here.

The paper is organized as follows. First of all, the new EoS and Riemann solver are derived. Secondly, the new EoS is verified and validated against real fluid thermodynamics. After that, the Riemann solver is verified and validated against ideal gas problems. Lastly, EoS and RS models are embedded into a one-dimensional Eulerian solver, and they are validated together against wave propagation in real fluids and in real fluid mixtures.

\section{Equation of State}

The thermodynamic states of the propellant in a liquid rocket engine, might be very different depending on in which part of the engine it flows, spanning through liquid, vapor, gas and super-critical states. For such a reason, different models might be used in different subsystems. This is true even when dealing with different locations within the same combustion chamber.

Staring at the combustion chamber of cryogenic liquid rocket engines, one might observe that both the fuel and oxidizer are often above their critical pressure. Concerning their temperature and density, the oxidizer is generally injected at a cryogenic temperature as it comes from the associated tank, and possibly the pump; consequently, the temperature is below its critical temperature, and the density is about that of its condensed state. On the other hand, fuel is injected in a gasified state since it comes from the cooling jacket, and its temperature is often higher than its critical value. Downstream of the mixture ignition location, the temperature becomes significantly high, and higher than the mixture critical temperature.

For the mentioned reasons, only the oxidizer is chosen to be treated as a real fluid, while fuel and combustion products are assumed to behave as ideal gases. Such an assumption allows to significantly simplify the EoS formulation.

So, starting from the original formulation by Kim et al. [16], we have the following:

$$
p\left(\rho, T, \chi_{i}\right)=\frac{\rho R_{u} T}{\mathfrak{w}-b \rho}-\frac{a \alpha(T) \rho^{2}}{\left(\mathfrak{w}+\delta_{1} \rho b\right)\left(\mathfrak{w}+\delta_{2} \rho b\right)}
$$

where $\rho, p, T, \mathfrak{w}$ represent the density, pressure, temperature and molar weight of the mixture, respectively, while $R_{u}$ is the universal gas constant and $a \alpha(T), b, \delta_{1}, \delta_{2}$, are model parameters, reading as follows:

$$
\begin{gathered}
a \alpha(T):=\sum_{i=1}^{N} \sum_{j=1}^{N} \chi_{i} \chi_{j} a_{i j} \alpha_{i j}(T) \quad \text { where } a_{i j} \alpha_{i j}(T):=\sqrt{a_{i} a_{j} \alpha_{i}(T) \alpha_{j}(T)}\left(1-\kappa_{i j}\right) \\
b:=\sum_{i=1}^{N} \chi_{i} b_{i}
\end{gathered}
$$




$$
\delta_{1}:=\sum_{i=1}^{N} \chi_{i} \delta_{1, i}, \quad \delta_{2}:=\sum_{i=1}^{N} \chi_{i} \delta_{2, i}
$$

where $\chi_{i}$ and $\chi_{j}$ are molar fractions of species $i$ and $j$. As can be seen from Equation (2), parameters $\alpha_{i}$ are temperature dependent, while parameters $a_{i}$ and $b_{i}$ are constants depending only on the species. Concerning parameters $\delta$, they are actually switches capable of making the cubic law to assume the shape of Soave-Redlich-Kwong [6] (SRK), Peng-Robinson [7] (PR) or Redlich-Kwong-Peng-Robinson [8] (RK-PR) EoSs. Each of the model parameters is shown in Table 1.

As previously mentioned, such a formulation is further simplified in order to consider only the oxidizer species as a real fluid, while fuel and combustion product mixtures are treated as ideal ones. It has to be noted that considering a species as an ideal one means assuming that it has an extremely high critical pressure and an extremely low critical temperature with respect to the operating pressure and temperature. This condition can be expressed by setting the coefficients $a$ and $b$ to zero (see Table 1 ).

In this sense, manipulating Equations (1) and (2) yields the following:

$$
p\left(\rho, T, \chi_{i}\right)=\frac{\rho R_{u} T}{\mathfrak{w}-\chi_{o} b_{o} \rho}-\frac{\chi_{o}^{2} a_{o} \alpha_{o}(T) \rho^{2}}{\left(\mathfrak{w}+\delta_{1} \rho \chi_{o} b_{o}\right)\left(\mathfrak{w}+\delta_{2} \rho \chi_{o} b_{o}\right)}
$$

where the mixture molecular weight $(\mathfrak{w})$ is expressed as follows:

$$
\mathfrak{w}:=\sum_{i} \chi_{i} w_{i}
$$

and where subscript $o$ stands for oxidizer, while the molar fractions and molar weight of the $i$-th species are expressed as $\chi_{i}$ and $w_{i}$.

\section{Other Thermodynamic Quantities}

A complete determination of the thermodynamic quantities can be found in [21]. The general expression of internal energy $(e)$ is the following:

$$
e\left(\rho, T, \chi_{i}\right)=e_{0}(T)+\int_{0}^{\rho}\left[\frac{p\left(\rho, T, \chi_{i}\right)}{\rho^{2}}-\frac{T}{\rho^{2}}\left(\frac{\partial p}{\partial T}\right)_{\rho, \chi_{i}}\right] \mathrm{d} \rho
$$

where subscript 0 represents the ideal quantities evaluated at a sufficiently rarefied reference state, in which ideal gas assumptions hold. Its derivative with respect to temperature, namely specific heat at constant volume $\left(c_{v}\right)$, reads as follows:

$$
c_{v}\left(\rho, T, \chi_{i}\right):=\left(\frac{\partial e}{\partial T}\right)_{\rho, \chi_{i}}=c_{v, 0}(T)+\int_{0}^{\rho}\left[-\frac{T}{\rho^{2}}\left(\frac{\partial^{2} p}{\partial T^{2}}\right)_{\rho, \chi_{i}}\right] \mathrm{d} \rho
$$

while specific heat at constant pressure is expressed as follows:

$$
c_{p}\left(\rho, T, \chi_{i}\right):=c_{v}\left(\rho, T, \chi_{i}\right)+\frac{T}{\rho^{2}} \frac{\left(\frac{\partial p}{\partial T}\right)_{\rho, \chi_{i}}^{2}}{\left(\frac{\partial p}{\partial \rho}\right)_{T, \chi_{i}}}
$$

Those quantities suffice to calculate the speed of sound, which is an important parameter in the present framework. It reads as follows:

$$
a\left(\rho, T, \chi_{i}\right):=\frac{c_{p}\left(\rho, T, \chi_{i}\right)}{c_{v}\left(\rho, T, \chi_{i}\right)}\left(\frac{\partial p}{\partial \rho}\right)_{T, \chi_{i}}
$$

For the sake of completeness, enthalpy is expressed as the following:

$$
h\left(\rho, T, \chi_{i}\right):=e\left(\rho, T, \chi_{i}\right)+\frac{p\left(\rho, T, \chi_{i}\right)}{\rho}
$$


Table 1. Characteristic parameters of the three EoSs. Subscript " $c$ " stands for critical and, thus, refers to quantities evaluated at the species critical point.

\begin{tabular}{|c|c|c|c|}
\hline Parameter & SRK [6] & PR [7] & RK-PR [8] \\
\hline $\begin{array}{l}\delta_{1} \\
\delta_{2}\end{array}$ & $\begin{array}{c}1 \\
\left(1-\delta_{1}\right) /\left(1+\delta_{1}\right)\end{array}$ & $\begin{array}{c}0 \\
\left(1-\delta_{1}\right) /\left(1+\delta_{1}\right)\end{array}$ & $\begin{array}{c}d_{1}+d_{2}\left(d_{3}-1.168 Z_{c}\right)^{d_{4}}+d_{5}\left(d_{3}-1.168 Z_{c}\right)^{d_{6}} \\
\left(1-\delta_{1}\right) /\left(1+\delta_{1}\right)\end{array}$ \\
\hline$a$ & $0.42747 \frac{R_{u}^{2} T_{c}^{2}}{p_{c}}$ & $0.45724 \frac{R_{u}^{2} T_{c}^{2}}{p_{c}}$ & $\frac{d^{2}+3 d y+d+3 y^{2}-1}{(d+3 y-1)^{2}}\left(\frac{R_{u}{ }^{2} T_{c}^{2}}{p_{c}}\right)$ \\
\hline$\alpha$ & $\begin{array}{c}0.08664 \frac{R_{u} T_{c}}{p_{c}} \\
\left(1+S\left(1-\sqrt{\frac{T}{T_{c}}}\right)\right)^{2}\end{array}$ & $\begin{array}{c}0.07780 \frac{R_{u} T_{c}}{p_{c}} \\
\left(1+S\left(1-\sqrt{\frac{T}{T_{c}}}\right)\right)^{2}\end{array}$ & $\begin{array}{c}\frac{1}{d+3 y-1}\left(\frac{R_{u} T_{c}}{p_{c}}\right) \\
\left(\frac{3}{2+T / T_{c}}\right)^{k}\end{array}$ \\
\hline$S$ & $-0.15613 \omega^{2}+1.55171 \omega+0.48508$ & $-0.26992 \omega^{2}+1.54226 \omega+0.37464$ & - \\
\hline$k$ & - & - & $\omega^{2}\left(A_{0}+1.168 A_{1} Z_{c}\right)+\omega\left(B_{0}+1.168 B_{1} Z_{c}\right)+\left(C_{0}+1.168 C_{1} Z_{c}\right)$ \\
\hline$d$ & - & - & $\left(1+\delta_{1}^{2}\right) /\left(1+\delta_{1}\right)$ \\
\hline$y$ & - & - & $\sqrt[3]{\frac{4}{\delta_{1}+1}}+\sqrt[3]{2\left(\delta_{1}+1\right)}+1$ \\
\hline$d_{1}$ & - & - & 0.428363 \\
\hline$d_{2}$ & - & - & 18.496215 \\
\hline$d_{4}$ & - & - & 0.66 \\
\hline$d_{5}$ & - & - & 789.723105 \\
\hline$d_{6}$ & - & - & 2.512392 \\
\hline$A_{0}$ & - & - & 0.0017 \\
\hline$A_{1}$ & - & - & -2.4407 \\
\hline$B_{0}$ & - & - & 1.9681 \\
\hline$B_{1}$ & - & - & 7.4513 \\
\hline$C_{0}$ & - & - & -2.7238 \\
\hline$C_{1}$ & - & - & 12.5040 \\
\hline
\end{tabular}




\section{Derivation of the Riemann Solver}

In the first part of this section, the definition of the governing equations is given. In the second part, the derivation of the solver is carried out.

\subsection{Governing Equations}

The governing equations are the one-dimensional Eulerian equations with variable cross-section area. A three-species problem is considered, including oxidizer, fuel and combustion product mixtures. In particular, conservation laws are selected to preserve total, oxidizer and fuel mass. Deriving from [17], we have the following:

$$
\frac{\partial U(x, t)}{\partial t}+\frac{\partial F(U(x, t))}{\partial x}=S(U(x, t))
$$

where

$$
\begin{gathered}
U:=\left\{\rho, \rho u, \rho e_{0}, \rho Y_{0}, \rho Y_{f}\right\}^{T} \\
F:=\left\{\rho u, \rho u^{2}+p, \rho u h_{0}, \rho u Y_{0}, \rho u Y_{f}\right\}^{T} \\
S=-\frac{1}{A} \frac{D A}{D t}\left\{\rho, \rho u, \rho h_{0}, \rho Y_{0}, \rho Y_{f}\right\}^{T}, \quad \text { where } \frac{D A}{D t}:=\frac{\partial A}{\partial t}+u \frac{\partial A}{\partial x}
\end{gathered}
$$

and where $\rho, u, p, h_{0}$ and $e_{0}$ represent the flow density, velocity, pressure, total enthalpy and total internal energy, respectively, while $Y_{o}$ and $Y_{f}$ represent oxidizer and fuel mixtures mass fractions, and $A$ is the cross-section area.

One might have noticed that mass fractions $\left(Y_{i}\right)$ are used in the governing equations, while the EoS employs molar fractions $\left(\chi_{i}\right)$. In such a framework, dealing with a threespecies system, the following can be said:

$$
\begin{aligned}
& \sum_{i} Y_{i}=Y_{o}+Y_{f}+Y_{p}=1 \quad \Rightarrow \quad Y_{p}=1-Y_{o}-Y_{f} \\
& \sum_{i} \chi_{i}=\chi_{o}+\chi_{f}+\chi_{p}=1 \quad \Rightarrow \quad \chi_{p}=1-\chi_{o}-\chi_{f}
\end{aligned}
$$

and therefore, Equation (4) can be recast as the following:

$$
\mathfrak{w}=\sum_{i} \chi_{i} w_{i}=\chi_{o} w_{o}+\chi_{f} w_{f}+\chi_{p} w_{p}=\chi_{o} w_{o}+\chi_{f} w_{f}+\left(1-\chi_{o}-\chi_{f}\right) w_{p}
$$

Furthermore the link between molar and mass fraction can be cast as the following:

$$
\begin{aligned}
& Y_{o}=\frac{\chi_{o} w_{o}}{\mathfrak{w}}=\frac{\chi_{o} w_{o}}{\chi_{f} w_{f}+\chi_{o} w_{o}+w_{p}\left(1-\chi_{o}-\chi_{f}\right)} \\
& Y_{f}=\frac{\chi_{f} w_{f}}{\mathfrak{w}}=\frac{\chi_{o} w_{o}}{\chi_{f} w_{f}+\chi_{o} w_{o}+w_{p}\left(1-\chi_{o}-\chi_{f}\right)}
\end{aligned}
$$

and inverting

$$
\begin{aligned}
& \chi_{o}=\frac{w_{f} w_{p} Y_{o}}{w_{o} w_{p} Y_{f}+w_{f} w_{p} Y_{o}+w_{f} w_{o}\left(1-Y_{o}-Y_{f}\right)} \\
& \chi_{f}=\frac{w_{o} w_{p} Y_{f}}{w_{o} w_{p} Y_{f}+w_{f} w_{p} Y_{o}+w_{f} w_{o}\left(1-Y_{o}-Y_{f}\right)}
\end{aligned}
$$


so the derivatives in $\partial Y_{i}$ can be computed using the following chain rule:

$$
\frac{\partial \phi}{\partial Y_{i}}=\frac{\partial \phi}{\partial \chi_{i}} \frac{\partial \chi_{i}}{\partial Y_{i}}
$$

by differentiating Equation (15).

\subsection{Riemann Solver}

Even by reconstructing the flow field piece-wise linearly (or higher order), it yields to discontinuities at the intercells, as shown in Figure 1, i.e., to a Riemann Problem (RP). RPs can be solved in many different ways [17], provided that the solution is consistent with the adopted system of equations, even if approximated.

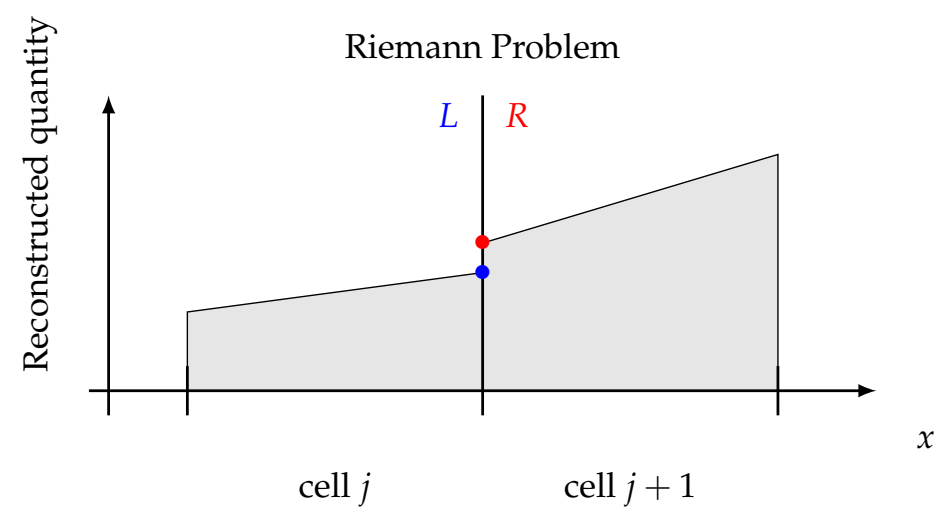

Figure 1. Riemann problem concept. Intercell discontinuity has to be solved consistently.

The Roe Solvers family [18-20] expresses the interface solution as the following:

$$
\begin{aligned}
U^{\mathrm{if}} & =\frac{U_{R}+U_{L}}{2}-\frac{1}{2} \tilde{\mathbf{R}} \frac{|\tilde{\mathbf{\Lambda}}|}{\tilde{\mathbf{\Lambda}}} \tilde{\mathbf{L}}\left(U_{R}-U_{L}\right) \\
F^{\mathrm{if}} & =\frac{F_{R}+F_{L}}{2}-\frac{1}{2} \tilde{\mathbf{R}}|\tilde{\mathbf{\Lambda}}| \tilde{\mathbf{L}}\left(U_{R}-U_{L}\right)
\end{aligned}
$$

where $\tilde{\mathbf{\Lambda}}, \tilde{\mathbf{L}}$ and $\tilde{\mathbf{R}}$ are the Jacobian eigenvalues and left and right eigenvectors matrices, respectively, and $U$ and $F$ are state and flux vectors, as in Equation (10). Superscript "if" stands for interface, while $L$ and $R$ stand for left and right sides of the interface, as shown in Figure 1. Tilded variables are computed at one specific average state, called the Roe state, researched here, around which the solution is linearized. It is worth to notice that Equation (17) - with no tildes-is always valid for any state, given that left and right states are close to each other. If they are not, the Roe state has to be constructed in such a way that jump relations are satisfied. Lastly, the original hyperbolicity of the system of equations has to be preserved as follows [17-20].

Summarizing, we have the following:

$$
\left\{\begin{array}{lcc}
\text { Hyperbolicity } & \Rightarrow & \text { Real eigenvalues } \\
\text { Consistency } & \Rightarrow & \tilde{\mathbf{J}} \text { such that } \tilde{\mathbf{J}}(U, U)=\mathbf{J}(U) \\
\text { Jump relations } & \Rightarrow & F_{L}-F_{R}=\tilde{\mathbf{J}}\left(U_{L}-U_{R}\right)
\end{array}\right.
$$

Since cross-section area variations can be treated as source terms, as can be seen in Equations (10) and (11), the Riemann solver can be derived from the homogeneous 5-by-5 system of equations. 
Following developments in $[17,20]$ while selecting a different set of primitive variables $W$ as

$$
W:=\left\{\rho, u, T, Y_{o}, Y_{f}\right\}^{T}
$$

a change of coordinates causes the homogeneous system (10) to become the following:

$$
\begin{array}{r}
\frac{\partial U}{\partial W} \frac{\partial W}{\partial t}+\frac{\partial F}{\partial U} \frac{\partial U}{\partial W} \frac{\partial W}{\partial x}=0 \\
\frac{\partial W}{\partial t}+\left(\frac{\partial U}{\partial W}\right)^{-1} \frac{\partial F}{\partial U} \frac{\partial U}{\partial W} \frac{\partial W}{\partial x}=0 \quad \Rightarrow \\
\frac{\partial W}{\partial t}+\left(\frac{\partial U}{\partial W}\right)^{-1} \frac{\partial F}{\partial W} \frac{\partial W}{\partial x}=0
\end{array}
$$

thus, a new Jacobian matrix $\mathbf{K}$ is obtained, in the new set of coordinates $W$, as the following:

$$
\mathbf{K}:=\left(\frac{\partial U}{\partial W}\right)^{-1} \frac{\partial F}{\partial W}
$$

By developing computations, and using the compact notation for derivatives $\left(\phi_{\psi}:=\partial \phi / \partial \psi\right)$, matrices become the following:

$$
\frac{\partial U}{\partial W}=\left(\begin{array}{ccccc}
1 & 0 & 0 & 0 & 0 \\
u & \rho & 0 & 0 & 0 \\
\rho e_{\rho}+e+\frac{u^{2}}{2} & \rho u & \rho e_{T} & \rho e_{Y_{o}} & \rho e_{Y_{f}} \\
Y_{o} & 0 & 0 & \rho & 0 \\
Y_{f} & 0 & 0 & 0 & \rho
\end{array}\right)
$$

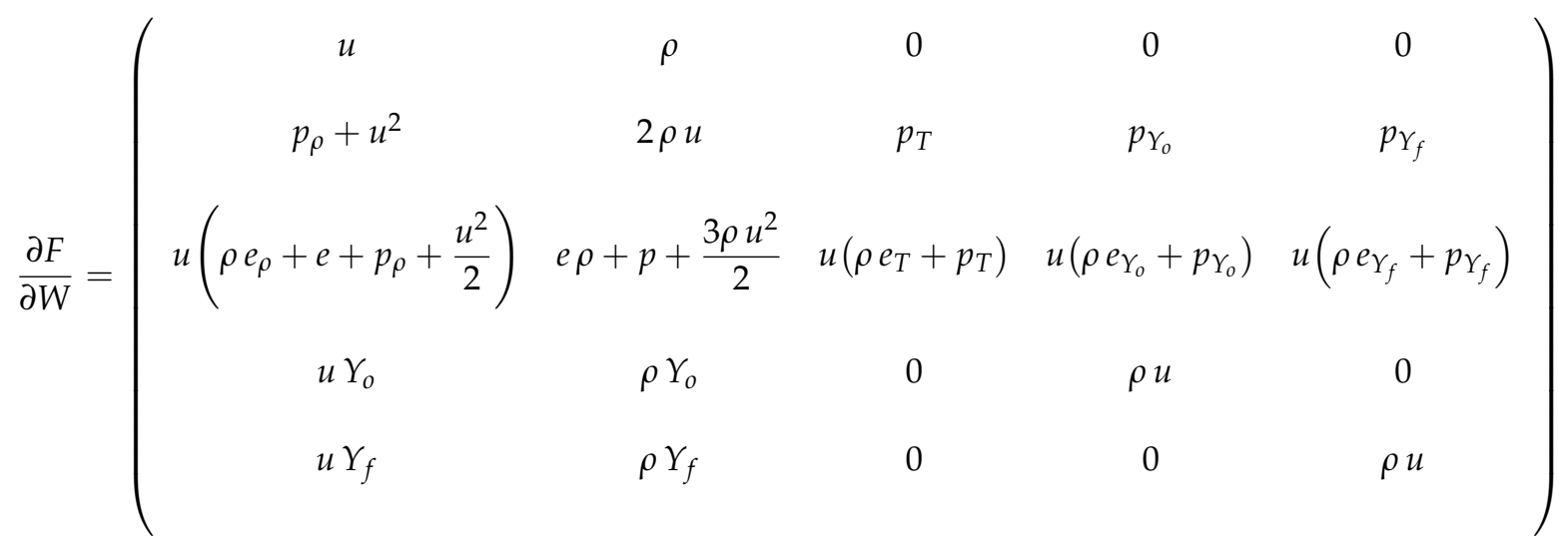

and thus, the Jacobian $\mathbf{K}$ reads as the following: 


$$
\mathbf{K}=\left(\begin{array}{ccccc}
u & \rho & 0 & 0 & 0 \\
\frac{p_{\rho}}{\rho} & u & \frac{p_{T}}{\rho} & \frac{p_{Y_{o}}}{\rho} & \frac{p_{Y_{f}}}{\rho} \\
0 & \frac{p-\rho^{2} e_{\rho}}{\rho e_{T}} & u & 0 & 0 \\
0 & 0 & 0 & u & 0 \\
0 & 0 & 0 & 0 & u
\end{array}\right)
$$

Computing eigenvalues yields the following:

$$
\operatorname{diag}(\boldsymbol{\Lambda})=\{u, u, u, u-a, u+a\}, \quad \text { where } a^{2}:=\frac{1}{e_{T}}\left(\frac{p p_{T}}{\rho^{2}}-e_{\rho} p_{T}\right)+p_{\rho}=\frac{c_{p}}{c_{v}} p_{\rho}
$$

while right and left eigenvectors, listed in matrices $\mathbf{R}_{\mathbf{K}}$ and $\mathbf{L}_{\mathbf{K}}$ by columns and rows, respectively, read as follows:

$$
\begin{aligned}
& \mathbf{R}_{\mathbf{K}}=\left(\begin{array}{ccccc}
-\frac{p_{Y_{f}}}{p_{\rho}} & -\frac{p_{Y_{o}}}{p_{\rho}} & -\frac{p_{T}}{p_{\rho}} & \frac{\rho^{2} e_{T}}{p-\rho^{2} e_{\rho}} & \frac{\rho^{2} e_{T}}{p-\rho^{2} e_{\rho}} \\
0 & 0 & 0 & \frac{a \rho e_{T}}{\rho^{2} e_{\rho}-p} & \frac{a \rho e_{T}}{p-\rho^{2} e_{\rho}} \\
0 & 0 & 1 & 1 & 1 \\
0 & 1 & 0 & 0 & 0 \\
1 & 0 & 0 & 0 & 0
\end{array}\right) \\
& \mathbf{L}_{\mathbf{K}}=\left(\begin{array}{ccccc}
0 & 0 & 0 & 0 & 1 \\
0 & 0 & 0 & 1 & 0 \\
\frac{p_{\rho}\left(p_{\rho}-a^{2}\right)}{a^{2} p_{T}} & 0 & \frac{p_{\rho}}{a^{2}} & \frac{p_{Y_{o}}\left(p_{\rho}-a^{2}\right)}{a^{2} p_{T}} & \frac{p_{Y_{f}}\left(p_{\rho}-a^{2}\right)}{a^{2} p_{T}} \\
\frac{p_{\rho}\left(a^{2}-p_{\rho}\right)}{2 a^{2} p_{T}} & \frac{\rho\left(p_{\rho}-a^{2}\right)}{2 a p_{T}} & \frac{1}{2}-\frac{p_{\rho}}{2 a^{2}} & \frac{p_{Y_{o}}\left(a^{2}-p_{\rho}\right)}{2 a^{2} p_{T}} & \frac{p_{Y_{f}}\left(a^{2}-p_{\rho}\right)}{2 a^{2} p_{T}} \\
\frac{p_{\rho}\left(a^{2}-p_{\rho}\right)}{2 a^{2} p_{T}} & \frac{\rho\left(a^{2}-p_{\rho}\right)}{2 a p_{T}} & \frac{1}{2}-\frac{p_{\rho}}{2 a^{2}} & \frac{p_{Y_{o}}\left(a^{2}-p_{\rho}\right)}{2 a^{2} p_{T}} & \frac{p_{Y_{f}}\left(a^{2}-p_{\rho}\right)}{2 a^{2} p_{T}}
\end{array}\right)
\end{aligned}
$$

For convenience, it is possible to recast the last condition in Equation (18) as the following:

$$
\left\{\begin{array}{l}
\Delta U=\tilde{\mathbf{H}} \Delta W \\
\Delta F=\tilde{\mathbf{A}} \Delta W
\end{array}\right.
$$

where

$$
\Delta W=\left\{\Delta \rho, \Delta u, \Delta T, \Delta Y_{o}, \Delta Y_{f}\right\}^{T}
$$




$$
\begin{gathered}
\Delta U=\left\{\Delta \rho, \Delta(\rho u), \Delta\left(\rho e_{0}\right), \Delta\left(\rho Y_{o}\right), \Delta\left(\rho Y_{f}\right)\right\}^{T} \\
\Delta F=\left\{\Delta(\rho u), \Delta\left(\rho u^{2}+p\right), \Delta\left(\rho u h_{0}\right), \Delta\left(\rho u Y_{o}\right), \Delta\left(\rho u Y_{f}\right)\right\}^{T}
\end{gathered}
$$

and where symbol $\Delta$ represents left-to-right quantity differences $\left(\Delta \phi:=\phi_{L}-\phi_{R}\right)$, and where matrices $\tilde{\mathbf{H}}, \tilde{\mathbf{A}}, \tilde{\mathbf{L}_{\mathbf{K}}}$ and $\tilde{\mathbf{R}_{\mathbf{K}}}$ are assumed to have the form of their untilded counterparts, in order to assure hyperbolicity. $\tilde{\mathbf{H}}$ and $\tilde{\mathbf{A}}$ read as the following:

$$
\tilde{\mathbf{H}}:=\widetilde{\left(\frac{\partial U}{\partial W}\right)}=\left(\begin{array}{ccccc}
1 & 0 & 0 & 0 & 0 \\
\tilde{u} & \tilde{\rho} & 0 & 0 & 0 \\
\tilde{\rho} \tilde{e}_{\rho}+\tilde{e}+\frac{\tilde{u}^{2}}{2} & \tilde{\rho} \tilde{u} & \tilde{\rho} \tilde{e}_{T} & \tilde{\rho} \tilde{e}_{Y_{o}} & \tilde{\rho} \tilde{e}_{Y_{f}} \\
\tilde{Y}_{o} & 0 & 0 & \tilde{\rho} & 0 \\
\tilde{Y}_{f} & 0 & 0 & 0 & \tilde{\rho}
\end{array}\right)
$$

$$
\tilde{\mathbf{A}}:=\widetilde{\left(\frac{\partial F}{\partial W}\right)}=\left(\begin{array}{ccccc}
\tilde{u} & \tilde{\rho} & 0 & 0 & 0 \\
\tilde{p}_{\rho}+\tilde{u}^{2} & 2 \tilde{\rho} \tilde{u} & \tilde{p}_{T} & \tilde{p}_{Y_{o}} & \tilde{p}_{Y_{f}} \\
\tilde{u}\left(\tilde{\rho} \tilde{e}_{\rho}+\tilde{e}+\tilde{p}_{\rho}\right)+\frac{\tilde{u}^{3}}{2} & \tilde{e} \tilde{\rho}+\tilde{p}+\frac{3 \tilde{\rho} \tilde{u}^{2}}{2} & \tilde{u}\left(\tilde{\rho} \tilde{e}_{T}+\tilde{p}_{T}\right) & \tilde{u}\left(\tilde{\rho} \tilde{e} Y_{o}+\tilde{p} Y_{o}\right) & \tilde{u}\left(\tilde{\rho} \tilde{e}_{Y_{f}}+\tilde{p}_{Y_{f}}\right) \\
\tilde{u} \tilde{Y}_{o} & \tilde{\rho} \tilde{Y}_{o} & 0 & \tilde{\rho} \tilde{u} & 0 \\
\tilde{u} \tilde{Y}_{f} & \tilde{\rho} \tilde{Y}_{f} & 0 & 0 & \tilde{\rho} \tilde{u}
\end{array}\right)
$$

Matrix $\tilde{\mathbf{H}}$ is the change-of-coordinates matrix between $U$ and $W$; thus, the following holds:

$$
\begin{gathered}
\tilde{\mathbf{L}}=\tilde{\mathbf{L}_{\mathbf{K}}} \tilde{\mathbf{H}}^{-1} \\
\tilde{\mathbf{R}}=\tilde{\mathbf{H}} \tilde{\mathbf{R}_{\mathbf{K}}}
\end{gathered}
$$

Solving system (28) for a consistent (in the sense of Equation (18)) set of averaged variables leads to the Roe state.

Gathering all the unknowns of system (28), considering also Equation (25), results in fourteen unknowns, namely the following:

$$
\tilde{Y}=\left\{\tilde{\rho}, \tilde{u}, \tilde{p}, \tilde{Y}_{o}, \tilde{Y}_{f}, \tilde{e}, \tilde{p}_{\rho}, \tilde{p}_{T}, \tilde{p}_{Y_{o}}, \tilde{p}_{Y_{f}}, \tilde{e}_{\rho}, \tilde{e}_{T}, \tilde{e}_{Y_{o}}, \tilde{e}_{Y_{f}}\right\}^{T}
$$

while system (28) employs ten equations. They read as follows:

$$
\begin{gathered}
\Delta \rho=\Delta \rho \\
\Delta(\rho u)=\Delta u \tilde{\rho}+\Delta \rho \tilde{u} \\
\Delta\left(\rho e_{0}\right)=\Delta T \tilde{\rho} \tilde{e} \tilde{e}_{T}+\Delta \rho\left(\tilde{\rho} \tilde{e}_{\rho}+\tilde{e}+\frac{\tilde{u}^{2}}{2}\right)+\Delta Y_{f} \tilde{\rho} \tilde{e}_{Y_{f}}+\Delta Y_{o} \tilde{\rho} \tilde{e}_{Y_{o}}+\Delta u \tilde{\rho} \tilde{u}
\end{gathered}
$$




$$
\begin{gathered}
\Delta\left(\rho Y_{o}\right)=\Delta Y_{o} \tilde{\rho}+\Delta \rho \tilde{Y}_{o} \\
\Delta\left(\rho Y_{f}\right)=\Delta Y_{f} \tilde{\rho}+\Delta \rho \tilde{Y}_{f} \\
\Delta(\rho u)=\Delta u \tilde{\rho}+\Delta \rho \tilde{u} \\
\Delta\left(\rho u^{2}+p\right)=\Delta T \tilde{p}_{T}+\Delta \rho\left(\tilde{p}_{\rho}+\tilde{u}^{2}\right)+\Delta Y_{f} \tilde{p}_{Y_{f}}+\Delta Y_{o} \tilde{p}_{Y_{o}}+2 \Delta u \tilde{\rho} \tilde{u} \\
\Delta\left(\rho u h_{0}\right)=\Delta T \tilde{u}\left(\tilde{\rho} \tilde{e} T+\tilde{p}_{T}\right)+\Delta \rho\left(\tilde{u}\left(\tilde{\rho} \tilde{\rho}_{\rho}+\tilde{e}+\tilde{p} \tilde{p}_{\rho}\right)+\frac{\tilde{u}^{3}}{2}\right)+ \\
+\Delta u\left(\tilde{e} \tilde{\rho}+\tilde{p}+\frac{3 \tilde{\rho} \tilde{u}^{2}}{2}\right)+\Delta Y_{f} \tilde{u}\left(\tilde{\rho} \tilde{Y}_{Y_{f}}+\tilde{p}_{Y_{f}}\right)+\Delta Y_{o} \tilde{u}\left(\tilde{\rho} \tilde{e} Y_{o}+\tilde{p} Y_{o}\right) \\
\Delta\left(\rho u Y_{o}\right)=\Delta Y_{o} \tilde{\rho} \tilde{u}+\Delta \rho \tilde{u} \tilde{Y}_{o}+\Delta u \tilde{\rho} \tilde{Y}_{o} \\
\Delta\left(\rho u Y_{f}\right)=\Delta Y_{f} \tilde{\rho} \tilde{u}+\Delta \rho \tilde{u} \tilde{Y}_{f}+\Delta u \tilde{\rho} \tilde{Y}{ }_{f}
\end{gathered}
$$

It is worth to notice that there are more unknowns than equations in the problem. Moreover, Equation (34a) is automatically satisfied, while Equation (34b,f), is redundant. Furthermore, as anticipated, the eight pressure and energy partial derivatives appear only in $(34 \mathrm{c}, \mathrm{g}, \mathrm{h})$. For those reasons, the system is not uniquely determined and some additional modeling is required, as expected [20].

\subsubsection{Density and Velocity}

Following the original formulation [20], density is consistently assumed to be the following:

$$
\tilde{\rho}:=\sqrt{\rho_{L} \rho_{R}}
$$

and keeping in mind that for a finite difference, the following equality holds:

$$
\Delta(\phi \cdot \psi)=\bar{\phi} \Delta \psi+\bar{\psi} \Delta \phi
$$

where

$$
\begin{aligned}
\Delta \psi & :=\psi_{L}-\psi_{R} \\
\bar{\psi} & :=\frac{\psi_{L}+\psi_{R}}{2}
\end{aligned}
$$

Equation (34f) can be recast as the following:

$$
\rho_{L} u_{L}+\tilde{\rho} u_{R}+\rho_{R} \tilde{u}=\rho_{R} u_{R}+\tilde{\rho} u_{L}+\rho_{L} \tilde{u}
$$

where substituting Equation (35) allows to solve for $\tilde{u}$ as the following:

$$
\tilde{u}=\frac{\sqrt{\rho_{L}} u_{L}+\sqrt{\rho_{R}} u_{R}}{\sqrt{\rho_{L}}+\sqrt{\rho_{R}}}
$$

\subsubsection{Mass Fractions}

Substituting quantities obtained so far into Equation $(34 \mathrm{~d}, \mathrm{e})$ gives the following: 


$$
\begin{gathered}
\tilde{Y}_{o}=\frac{\sqrt{\rho_{L}} Y_{o, L}+\sqrt{\rho_{R}} Y_{o, R}}{\sqrt{\rho_{L}}+\sqrt{\rho_{R}}} \\
\tilde{Y}_{f}=\frac{\sqrt{\rho_{L}} Y_{f, L}+\sqrt{\rho_{R}} Y_{f, R}}{\sqrt{\rho_{L}}+\sqrt{\rho_{R}}}
\end{gathered}
$$

\subsubsection{Total Internal Energy}

The total internal energy $\left(e_{0}\right)$ can be deduced from Equation (34c), as follows. Being that $e_{0}$ is the function of

$$
e_{0}:=e_{0}\left(\rho, T, Y_{o}, Y_{f}, u\right)
$$

its total differential and partial derivatives read as the following:

$$
\begin{aligned}
\mathrm{d} e_{0}=\frac{\partial e_{0}}{\partial \rho} \mathrm{d} \rho+\frac{\partial e_{0}}{\partial T} \mathrm{~d} T+\frac{\partial e_{0}}{\partial Y_{o}} \mathrm{~d} Y_{o}+\frac{\partial e_{0}}{\partial Y_{f}} \mathrm{~d} Y_{f}+\frac{\partial e_{0}}{\partial u} \mathrm{~d} u \\
\frac{\partial e_{0}}{\partial \rho}=\frac{\partial e}{\partial \rho} \\
\frac{\partial e_{0}}{\partial T}=\frac{\partial e}{\partial T} \\
\frac{\partial e_{0}}{\partial Y_{o}}=\frac{\partial e}{\partial Y_{o}} \\
\frac{\partial e_{0}}{\partial Y_{f}}=\frac{\partial e}{\partial Y_{f}} \\
\frac{\partial e_{0}}{\partial u}=u
\end{aligned}
$$

thus, Equation (34c) can be recast as the following:

$$
\Delta \rho e_{0}=\Delta \rho\left(\tilde{e}+\frac{\tilde{u}^{2}}{2}\right)+\tilde{\rho}\left(\Delta \rho \tilde{e}_{\rho}+\Delta T \tilde{e}_{T}+\Delta Y_{f} \tilde{e}_{Y_{f}}+\Delta Y_{o} \tilde{e} Y_{o}+\Delta u \tilde{u}\right)
$$

At this point, a new equation for the Roe' state is introduced on the basis of energy differential, and it reads as the following:

$$
\Delta e_{0}=\tilde{e}_{0, \rho} \Delta \rho+\tilde{e}_{0, T} \Delta T+\tilde{e}_{0, u} \Delta u+\tilde{e}_{0, Y_{f}} \Delta Y_{f}+\tilde{e}_{0, Y_{0}} \Delta Y_{o}
$$

Recasting again Equation (34c) using Equation (44) and defining $\tilde{e}_{0}$ as

$$
\tilde{e}_{0}:=\tilde{e}+\frac{\tilde{u}}{2}
$$

gives the following:

$$
\Delta e_{0} \rho+\Delta \rho e_{0}=\Delta e_{0} \tilde{\rho}+\Delta \rho \tilde{e}_{0}
$$

where solving for $\tilde{e}_{0}$ yields the following:

$$
\tilde{e}_{0}=\frac{\sqrt{\rho_{L}} e_{0, L}+\sqrt{\rho_{R}} e_{0, R}}{\sqrt{\rho_{L}}+\sqrt{\rho_{R}}}
$$


As a consequence, internal energy ( $\tilde{e})$ reads as the following:

$$
\tilde{e}=\tilde{e}_{0}-\frac{\tilde{u}^{2}}{2}=\frac{e_{L} \sqrt{\rho_{L}}+e_{R} \sqrt{\rho_{R}}}{\sqrt{\rho_{L}}+\sqrt{\rho_{R}}}+\frac{\sqrt{\rho_{L}} \sqrt{\rho_{R}}\left(u_{L}-u_{R}\right)^{2}}{2\left(\sqrt{\rho_{L}}+\sqrt{\rho_{R}}\right)^{2}}
$$

It is important to remark that such determination of internal energies is not unique. In fact, the usage of the finite difference decomposition, as in Equation (36), allows to solve

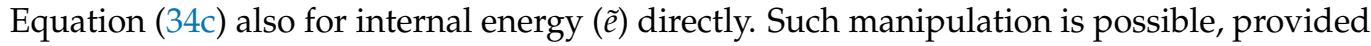
the inclusion of the counterpart of Equation (44), written for $\Delta \tilde{e}$. It ends up with the internal energy $(\tilde{e})$ having the form of Equation (46), while the total internal energy $\left(\tilde{e}_{0}\right)$ has the form of Equation (47), with opposite signs.

\subsection{Enthalpy}

Using exactly the same procedure as done for internal energy, it is possible to solve Equation (34h) for total specific enthalpy. The procedure is identical and thus synthesized for brevity. Introducing the definition of tilded, the total specific enthalpy is the following:

$$
\tilde{h}_{0}:=\tilde{h}+\frac{\tilde{u}^{2}}{2}:=\tilde{e}+\frac{\tilde{p}}{\tilde{\rho}}+\frac{\tilde{u}^{2}}{2}
$$

including the definition of enthalpy discrete differential $\left(\Delta h_{0}\right)$, as done for energy,

$$
\Delta h_{0}=\tilde{h}_{0, \rho} \Delta \rho+\tilde{h}_{0, T} \Delta T+\tilde{h}_{0, u} \Delta u+\tilde{h}_{0, Y_{f}} \Delta Y_{f}+\tilde{h}_{0, Y_{o}} \Delta Y_{o}
$$

and substituting it into Equation (34h) yields the following:

$$
\Delta(\rho u h 0)=\tilde{h}_{0}(\Delta u \tilde{\rho}+\Delta \rho \tilde{u})+\Delta h_{0} \tilde{\rho} \tilde{u}
$$

Solving for $\tilde{h}_{0}$ results in the following:

$$
\tilde{h}_{0}=\frac{h_{0, L} \sqrt{\rho L}+h_{0, R} \sqrt{\rho R}}{\sqrt{\rho L}+\sqrt{\rho R}}
$$

Specific enthalpy can be obtained from Equation (48): the result is analogous to the energy result.

In addition, concerning enthalpy, it is worth to note that Equation (34h) can be solved for total specific enthalpy $\left(\tilde{h}_{0}\right)$, specific enthalpy $(\tilde{h})$, or total enthalpy per unit volume $\left(\widetilde{\rho h_{0}}\right)$. Solving for $\widetilde{\rho h_{0}}$ yields a solution that is coincident with the one just showed $\left(\widetilde{\rho h_{0}} \equiv \tilde{\rho} \tilde{h}_{0}\right)$. On the other hand, solving for $\tilde{h}$ results in a completely different expression, that is more complicated and computationally intensive for all the quantities.

For the sake of simplicity and computational speed, Equations $(34 \mathrm{c}, \mathrm{h})$ are chosen to be solved for $\tilde{e}_{0}$ and $\tilde{h}_{0}$, respectively.

\subsection{Pressure}

Pressure is defined as the difference between $\tilde{h}_{0}$ and $\tilde{e}_{0}$ :

$$
\tilde{p}=\tilde{\rho}\left(\tilde{h_{0}}-\tilde{e_{0}}\right)
$$


yielding the following:

$$
\begin{array}{r}
\tilde{h}_{0}-\tilde{e}_{0}=\frac{\left(\frac{p_{L}}{\rho_{L}}\right) \sqrt{\rho_{L}}+\left(\frac{p_{R}}{\rho_{R}}\right) \sqrt{\rho_{R}}}{\sqrt{\rho_{L}}+\sqrt{\rho_{R}}}=\frac{\frac{p_{L}}{\sqrt{\rho_{L}}}+\frac{p_{R}}{\sqrt{\rho_{R}}}}{\sqrt{\rho_{L}}+\sqrt{\rho_{R}}} \quad \Rightarrow \\
\Rightarrow \quad \tilde{p}=\frac{p_{L} \sqrt{\rho_{R}}+p_{R} \sqrt{\rho_{L}}}{\sqrt{\rho_{L}}+\sqrt{\rho_{R}}}
\end{array}
$$

\subsection{Other Equations}

Equations in system (34) are all used, except Equation (34i,j,h). Concerning Equation (34i, $)$, they are identically satisfied by the quantities obtained so far. Concerning Equation (34g) on the other hand, it can be manipulated to obtain the following:

$$
\Delta\left(\rho u^{2}\right)+\Delta p=\Delta \rho \tilde{u}^{2}+2 \Delta u \tilde{\rho} \tilde{u}+\left(\tilde{p}_{\rho} \Delta \rho+\tilde{p}_{T} \Delta T+\tilde{p}_{Y_{f}} \Delta Y_{f}+\tilde{p}_{Y_{o}} \Delta Y_{o}\right)
$$

Such an equation can be identically satisfied, provided the inclusion of a discrete differential in tilde variables also for pressure as follows:

$$
\Delta p=\tilde{p}_{\rho} \Delta \rho+\tilde{p}_{T} \Delta T+\tilde{p}_{Y_{f}} \Delta Y_{f}+\tilde{p}_{Y_{o}} \Delta Y_{o}
$$

\subsection{Partial Derivatives of Pressure and Internal Energy}

There are eight quantities yet to be defined in the unknowns vector in Equation (33) and in system (34): they are pressure and internal energy partial derivatives. Since pressure derivatives appear only in their respective tilde differentials definitions, they need to be modeled.

Pressure partial derivatives are evaluated using the same form of pressure as Equation (53) (using the compact notation $p_{\psi}:=\partial p / \partial \psi$ ) as the following:

$$
\begin{aligned}
& \tilde{p}_{\rho}=\tilde{\rho} \frac{\left(\frac{p_{\rho, L}}{\sqrt{\rho_{L}}}+\frac{p_{\rho, R}}{\sqrt{\rho_{R}}}\right)}{\sqrt{\rho_{L}}+\sqrt{\rho_{R}}} \\
& \tilde{p}_{T}=\tilde{\rho} \frac{\left(\frac{p_{T, L}}{\sqrt{\rho_{L}}}+\frac{p_{T, R}}{\sqrt{\rho_{R}}}\right)}{\sqrt{\rho_{L}}+\sqrt{\rho_{R}}} \\
& \tilde{p}_{Y_{o}}=\tilde{\rho} \frac{\left(\frac{p_{Y_{o}, L}}{\sqrt{\rho_{L}}}+\frac{p_{Y_{o}, R}}{\sqrt{\rho_{R}}}\right)}{\sqrt{\rho_{L}}+\sqrt{\rho_{R}}} \\
& \begin{cases}\tilde{p}_{Y_{f}}=\frac{\Delta p-\tilde{p}_{\rho} \Delta \rho+\tilde{p}_{T} \Delta T-\tilde{p}_{u} \Delta u-\tilde{p}_{Y_{o}} \Delta Y_{o}}{\Delta Y_{f}} & \text { if } Y_{f, L} \neq Y_{f, R} \\
\tilde{p}_{Y_{f}}=\tilde{\rho} \frac{\left(\frac{p_{Y_{f}, L}}{\left.\sqrt{\rho_{L}}+\frac{p_{Y_{f}, R}}{\sqrt{\rho_{R}}}\right)}\right.}{\sqrt{\rho_{L}}+\sqrt{\rho_{R}}} & \text { if } Y_{f, L}=Y_{f, R}=Y_{f}\end{cases}
\end{aligned}
$$

where the first case of Equation (56d) is necessary to satisfy Equation (55). 
In the same way, energy partial derivatives are taken like the energy definition, Equation (47). They read as the following:

$$
\begin{gathered}
\tilde{e}_{\rho}=\frac{\sqrt{\rho_{L}} e_{\rho, L}+\sqrt{\rho_{R}} e_{\rho, R}}{\sqrt{\rho_{L}}+\sqrt{\rho_{R}}}+\langle u\rangle \\
\tilde{e}_{T}=\frac{\sqrt{\rho_{L}} e_{T, L}+\sqrt{\rho_{R}} e_{T, R}}{\sqrt{\rho_{L}}+\sqrt{\rho_{R}}}+\langle u\rangle \\
\tilde{e}_{Y_{o}}=\frac{\sqrt{\rho_{L}} e_{Y_{0}, L}+\sqrt{\rho_{R}} e_{Y_{0}, R}}{\sqrt{\rho_{L}}+\sqrt{\rho_{R}}}+\langle u\rangle \\
\frac{\partial \tilde{e}_{0}}{\partial \tilde{e}} \Delta Y_{f} \quad \text { if } Y_{f, L} \neq Y_{f, R} \\
\tilde{e}_{Y_{f}}=\frac{\Delta e_{0}-\tilde{e}_{0, \rho} \Delta \rho-\tilde{e}_{0, T} \Delta T-\tilde{e}_{0, u} \Delta u-\tilde{e}_{0, Y_{o}} \Delta Y_{o}}{\tilde{e}_{Y_{f}}=\frac{\sqrt{\rho_{L}} e_{Y_{f}, L}+\sqrt{\rho_{R}} e_{Y_{f}, R}}{\sqrt{\rho_{L}}+\sqrt{\rho_{R}}}+\langle u\rangle} \quad \text { if } Y_{f, L}=Y_{f, R}=Y_{f}
\end{gathered}
$$

where $\partial \tilde{e}_{0} / \partial \tilde{e}=1$, where

$$
\langle u\rangle:=\frac{\sqrt{\rho_{L}} \sqrt{\rho_{R}}\left(u_{L}-u_{R}\right)^{2}}{2\left(\sqrt{\rho_{L}}+\sqrt{\rho_{R}}\right)^{2}}
$$

and, again, where the first case of Equation (57d) is necessary to satisfy Equation (44).

\section{Verification and Validation}

The two models developed so far, i.e., the simplified cubic EoS for mixtures and the three-species Roe solver for generic gases, were implemented into an in-house onedimensional CFD solver. Such a solver employs finite volume first- and second-order Godunov-like numerical schemes.

The verification and validation processes follow different subsequent steps, which was also reported in [22]. First of all, the EoS subroutine is validated against the NIST database, by means of the refprop software [23], for both pure fluids and mixtures. Secondly, RS is validated against ideal gas Riemann problems. It has to be remarked that such a formulation of the Riemann solver is independent of any EoS. For such a reason, the ideal EoS, which was formerly coded in the solver and already validated in previous works (e.g., [24]), it was chosen as a first step of the RS validation. Lastly, the Riemann solver and the EoS are used in synergy to reproduce real gas Riemann problems and simple wave propagation in shock tubes.

Thermodynamic states investigated in this section are those representative of a typical oxidizer post and of a shear coaxial injector mixing zone. The reason for not investigating the hot-gas regime is justified by the fact that combustion products in the chamber core are assumed to behave as an ideal gas mixture as commonly assumed elsewhere $[25,26]$.

\subsection{EoS Validation}

Validation is performed using each of the three cubic EoS into which Equation (3) can be recast, namely SRK, PR and RK-PR. Density, internal energy and speed of sound are chosen as observables. 


\subsubsection{Pure Oxygen, Low Temperature}

Considering the aforementioned class of problems that will be faced with such a model, it is important to capture the oxidizer's real fluid behavior. Since the oxidizer comes directly from the tanks or pumps, the investigated temperature range is selected to be $50-400 \mathrm{~K}$, while pressure 6, 12 and $18 \mathrm{MPa}$. Molecular oxygen $\left(\mathrm{O}_{2}\right)$ is considered, its properties are listed in Table 2. Errors are estimated as the nondimensionalized L2-norms calculated between the EoS results and the NIST dataset, over the whole temperature range, for a single pressure value.

Table 2. Proprieties of molecular oxygen $\left(\mathrm{O}_{2}\right)$.

\begin{tabular}{lccc}
\hline Quantity & Symbol & Value & Unit \\
\hline Critical Temperature & $T_{c, o}$ & 154.581 & $\mathrm{~K}$ \\
Critical Pressure & $p_{c, o}$ & 5.043 & $\mathrm{MPa}$ \\
Critical Density & $\rho_{c, o}$ & 436.1 & $\mathrm{~kg} / \mathrm{m}^{3}$ \\
Critical Compressibility & $Z_{c, o}$ & 0.287925 & - \\
Acentric Factor & $\omega_{o}$ & 0.0222 & - \\
Molecular weight & $w_{o}$ & 31.999 & $\mathrm{~kg} / \mathrm{kmol}$ \\
\hline
\end{tabular}

Results of real $\mathrm{O}_{2}$ are reported in Figures 2 and 3, where the following can be noticed. First of all, supercritical oxygen behavior is qualitatively captured by each of the three EoSs. As the temperature turns higher, all the curves collapse onto the ideal ones, correctly. It appears that different quantities are affected by different levels of errors, e.g., PR EoS seems to show higher displacements on density and internal energy, while speed of sound behaves comparably with the other two EoS, especially at low pressures. Speed of sound is the quantity affected by the greater error with respect to NIST data, reaching an L2-norm error value close to $10 \%$ (see Figure 3c), and a local error value that is even higher, up to $20-25 \%$ depending on the EoS considered. Such an aspect is expected since it is known in the literature [16].

\subsubsection{Oxygen-Methane Mixture, Mid Temperature}

As a second validation test, the behavior of the EoS dealing with mixtures is assessed. An $\mathrm{O}_{2}-\mathrm{CH}_{4}$ mixture with an $\mathrm{O} / \mathrm{F}$ ratio of 3.4 is selected, which is a possible value of interest in liquid rocket engine chambers. It has to be recalled here that methane is treated as an ideal species, while oxygen as a real one. Differently from the oxidizer, fuel comes from the cooling jacket and thus, its temperature might be quite high. For such a reason, the temperature range of $300-1000 \mathrm{~K}$ is selected, as it is an interesting range to be investigated. Pressure values are 6,12 and $18 \mathrm{MPa}$. Again, errors are estimated as the nondimensionalized L2-norms calculated between the EoS results and the NIST dataset over the whole temperature range for a single pressure value.

The results of the real $\mathrm{O}_{2}-\mathrm{CH}_{4}$ mixture are available in Figures 4 and 5. It clearly appears in the plots that the real and ideal behaviors are quite close to each other, at those thermodynamic states. The quantity that shows a slightly higher disagreement is again the speed of sound. Concerning the EoSs, they show quite a coincident behavior, in this test. Errors lie between $0.5 \%$ and $5.5 \%$, depending on the EoS and on the observable. 


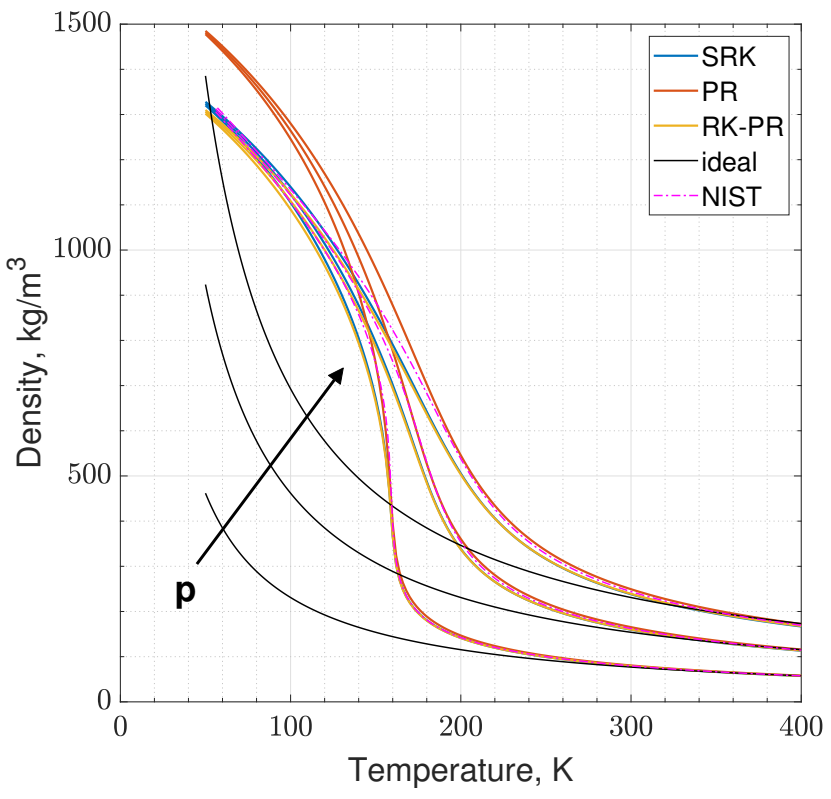

(a) Density.

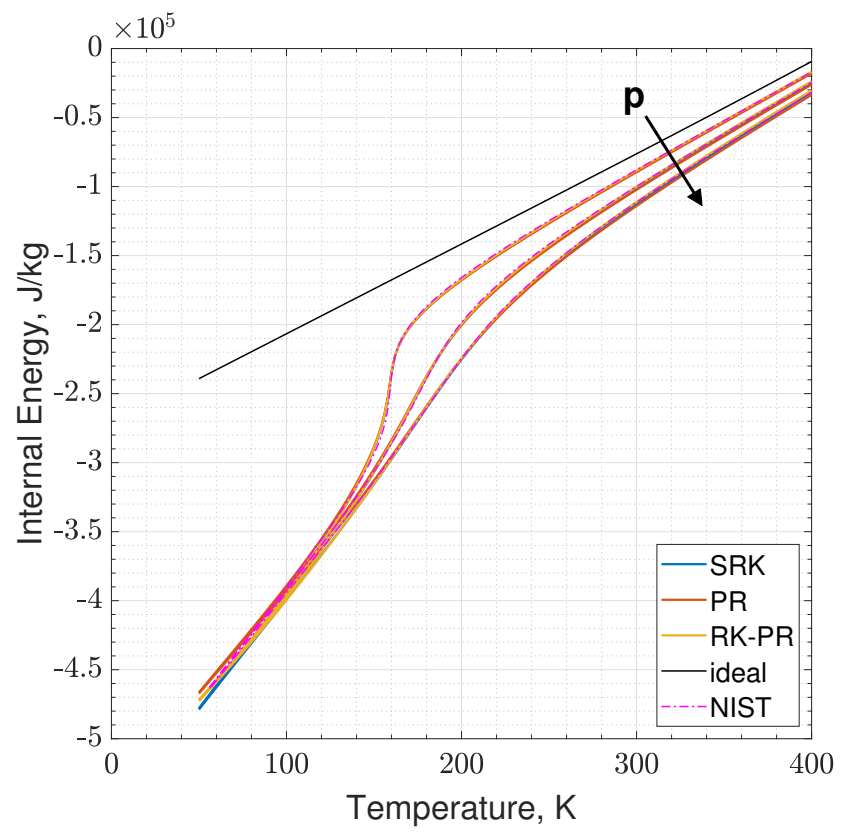

(b) Internal energy.

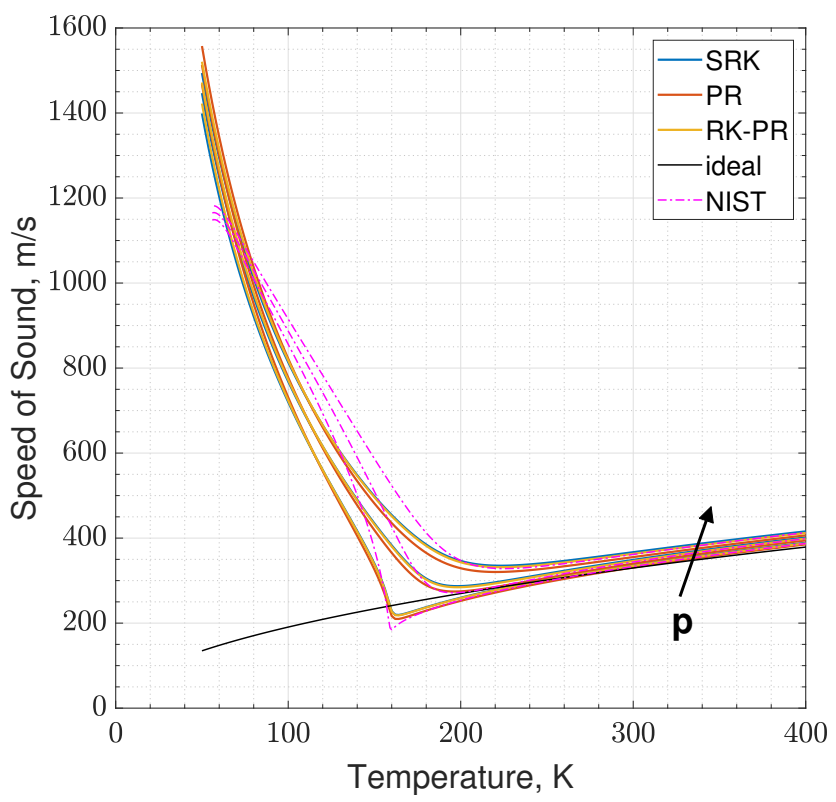

(c) Speed of sound.

Figure 2. Comparison of the present EoS in its SRK, PR and RK-PR modes with refprop data and ideal behavior. Species is pure $\mathrm{O}_{2}$. Temperature range is $50-400 \mathrm{~K}$. Pressure values are 6, 12 and $18 \mathrm{MPa}$. 


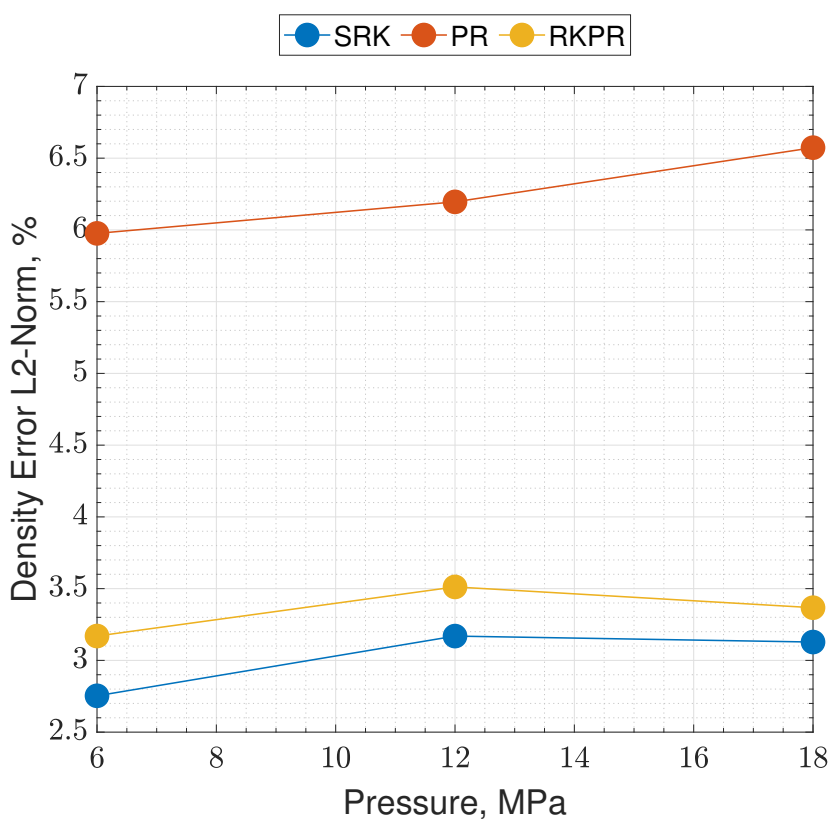

(a) Density.

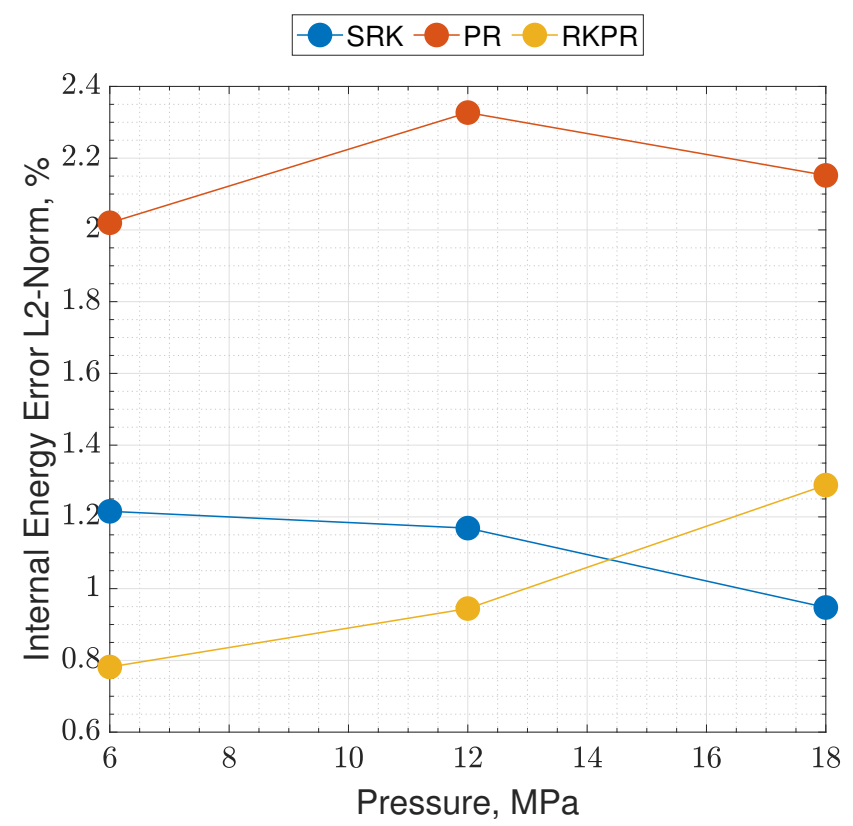

(b) Internal energy.

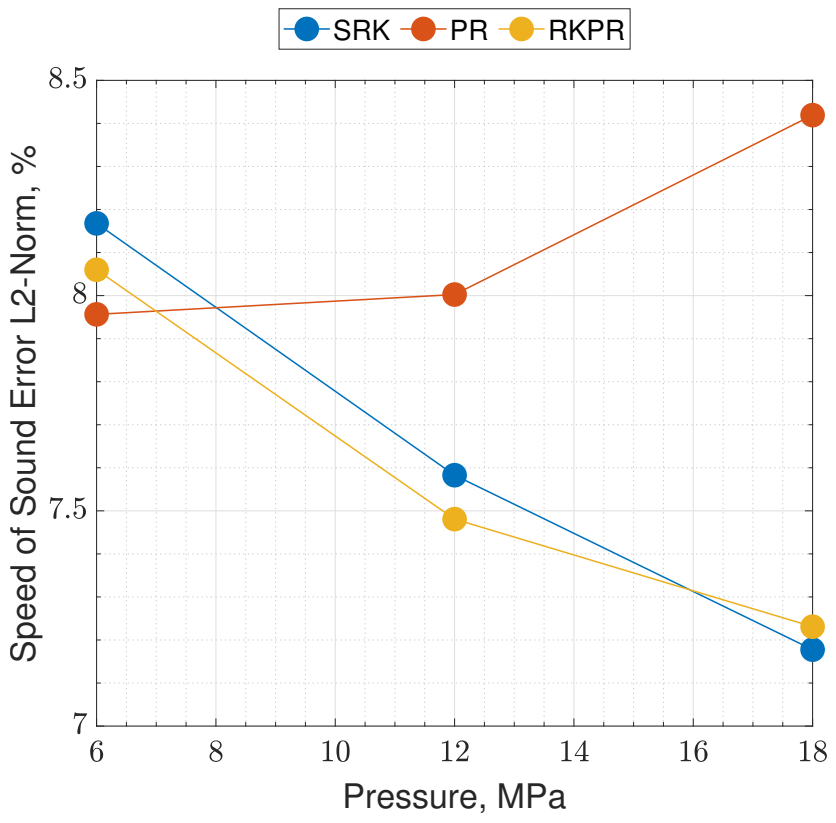

(c) Speed of sound.

Figure 3. Percent errors of the present EoS in its SRK, PR and RK-PR operative modes with respect to refprop data. Species is pure $\mathrm{O}_{2}$. Errors are estimated as the nondimensionalized L2-norms calculated between the EoS results and the NIST dataset, over the whole temperature range, for a single pressure value. 


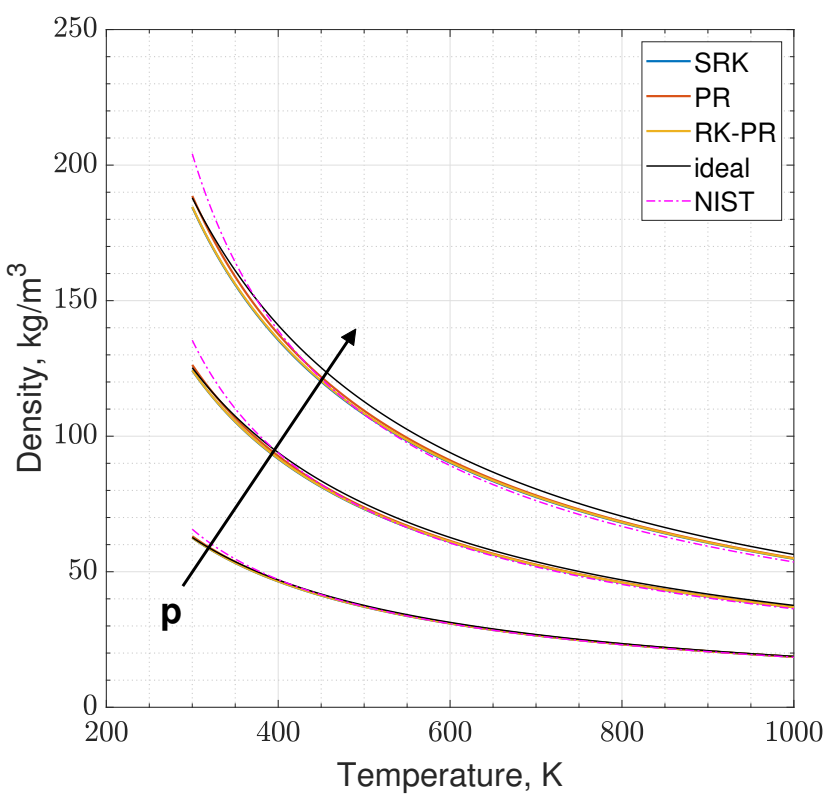

(a) Density.

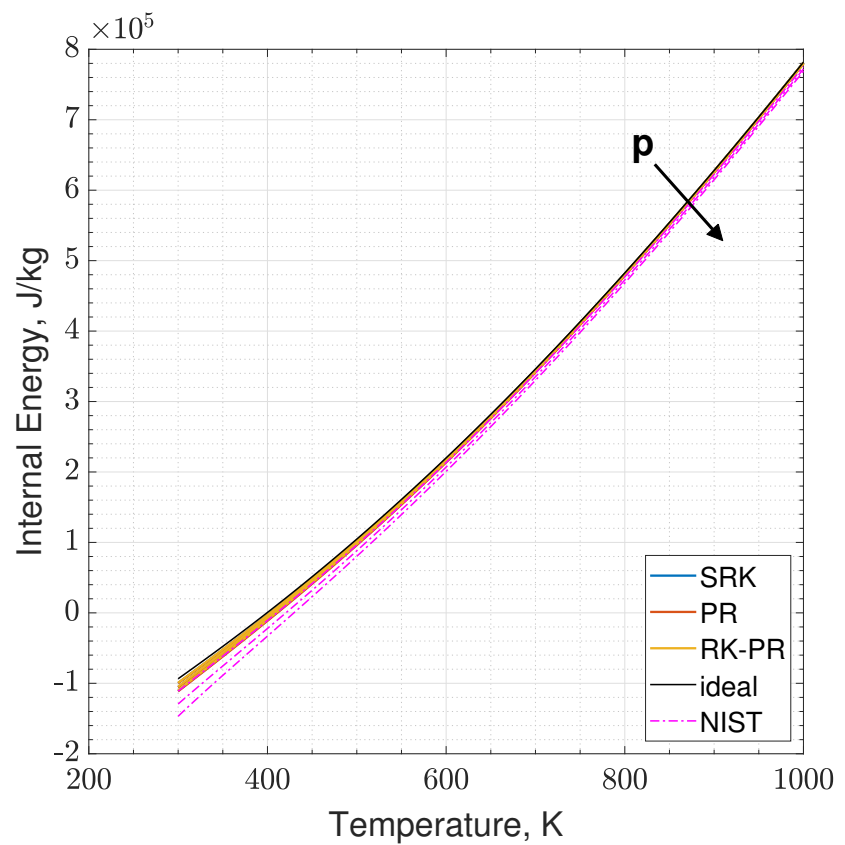

(b) Internal energy.

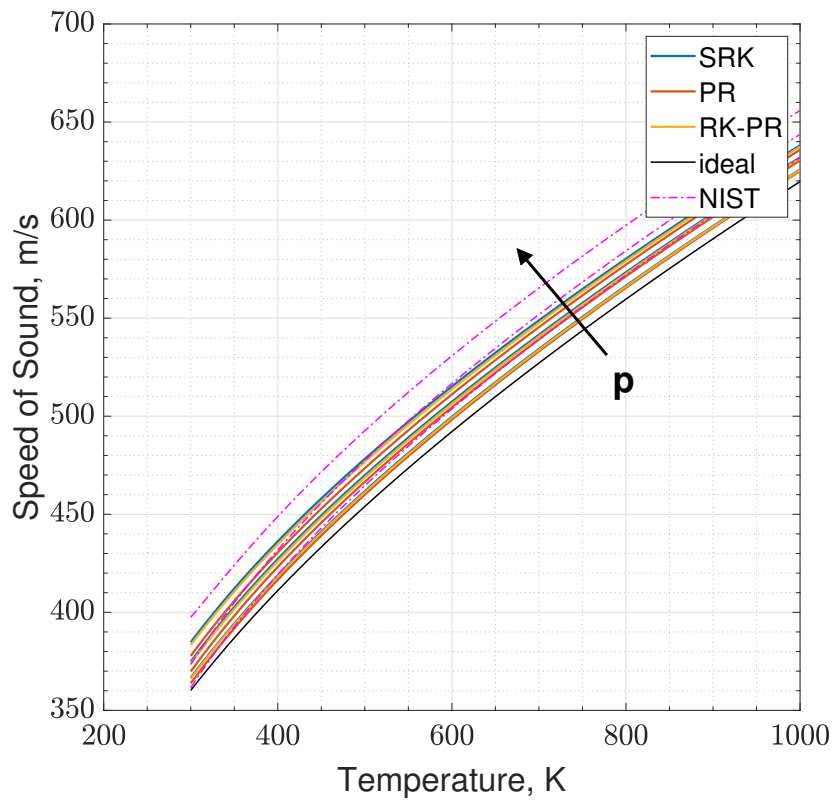

(c) Speed of sound.

Figure 4. Comparison of the present EoS in its SRK, PR and RK-PR modes with refprop data and ideal behavior. Mixture is $\mathrm{O}_{2}-\mathrm{CH}_{4}$ with $\mathrm{O} / \mathrm{F}=3.4$. Temperature range is $300-1000 \mathrm{~K}$. Pressure values are 6,12 and $18 \mathrm{MPa}$. 


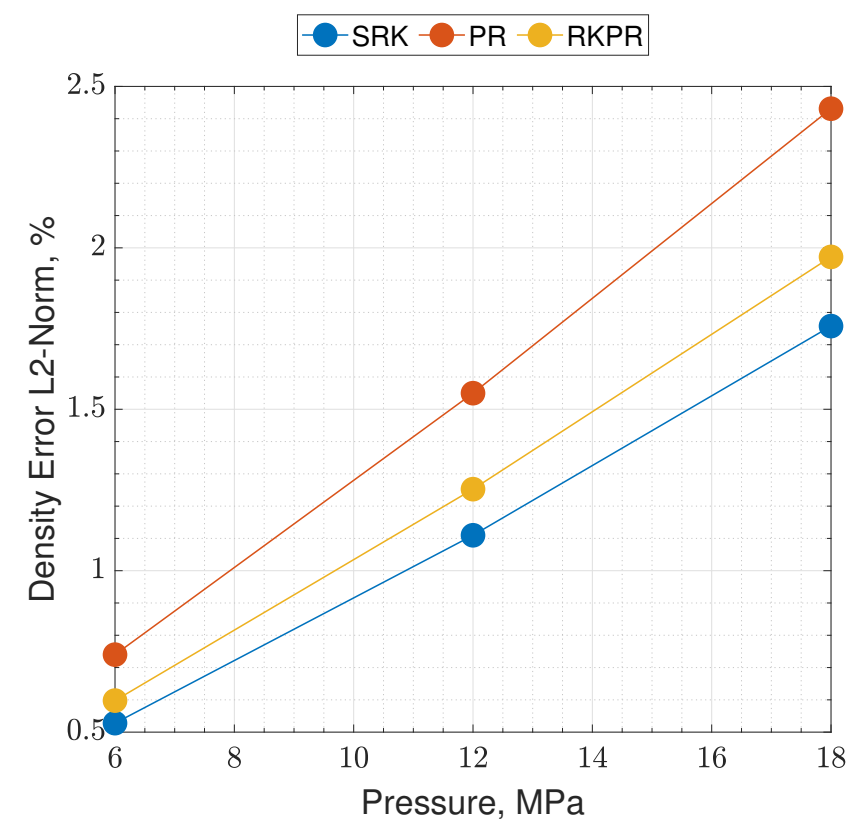

(a) Density.

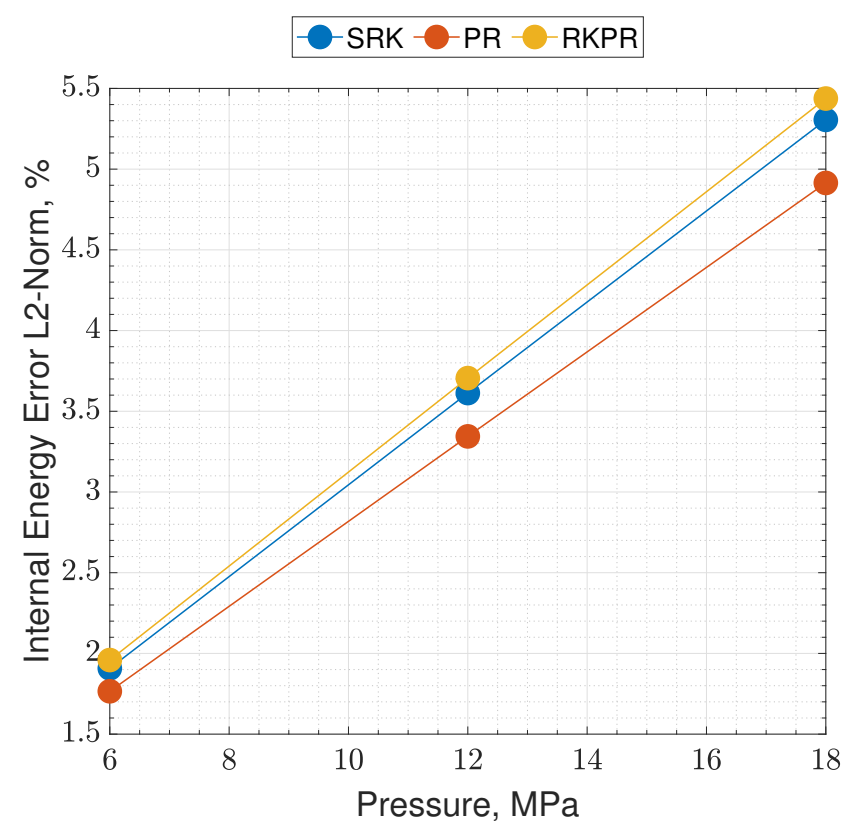

(b) Internal energy.

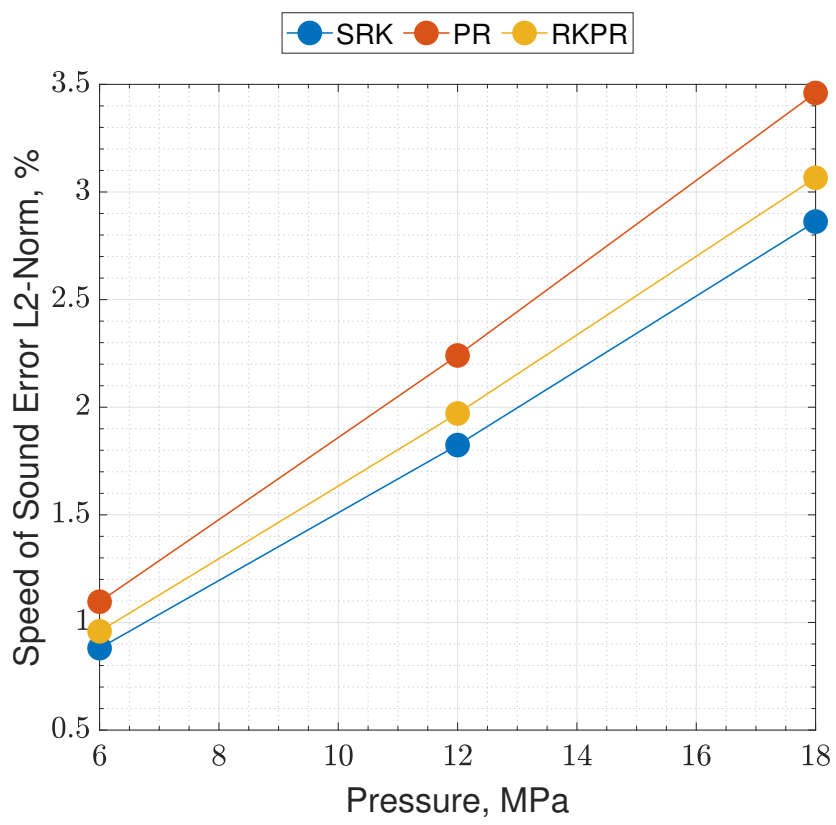

(c) Speed of sound.

Figure 5. Percent errors of the present EoS in its SRK, PR and RK-PR operative modes with respect to refprop data. Mixture is $\mathrm{O}_{2}-\mathrm{CH}_{4}$ with $\mathrm{O} / \mathrm{F}=3.4$. Errors are estimated as the nondimensionalized L2-norms calculated between the EoS results and the NIST dataset over the whole temperature range for a single pressure value. 


\subsubsection{Ideal Gas Riemann Problems}

The next step in the validation process involves the Riemann solver. For such a reason, two ideal gas Riemann problems are computed. The reason for such a choice lies in the fact that the ideal EoS was thoroughly validated in previous works (e.g., [24]), and in the fact that the solution of the ideal gas Riemann problems can be easily computed. The solver integrates the full conservative version of conservation laws listed in Equation (10) over a domain discretized in one thousand cells. Even if the number of cells seems high for a plain Riemann problem, it has to be pointed out that the physical length of the domain is $200 \mathrm{~m}$; thus, $\Delta x$ is $20 \mathrm{~cm}$. Such a high space span introduces significant numerical errors. The reason for choosing a domain this wide is justified by the fact that classical non-dimensional Riemann problems do not fit well with gas properties polynomials, so they are treated dimensionally, for convenience. The two selected problems are the Sod problem and the double symmetric expansion problem. The former describes the typical shock tube problem in which the two halves of the domain are separated by a diaphragm and are pressurized differently. In particular, the left-to-right side pressure ratio is 10, while the density ratio is 8 . The velocity is uniformly null over the entire domain. As the diaphragm is removed, a rarefaction wave is generated leftwards, which is almost sonic, whilst a contact discontinuity and shock wave begin traveling rightwards (RCS (rarefaction-contact-shock) solution). The latter, the double symmetric expansion problem, considers the two zones as having uniform pressure and density but equal and opposite velocities as the initial condition. In particular, the left-hand side velocity is negative, while the right-hand side value is positive. The anticipated solution is characterized by two expansion waves traveling in both directions symmetrically, with quiescent low pressure fluid in the middle (RNR (rarefaction-null-rarefaction) solution). The initial conditions are summarized in Table 3.

Table 3. Ideal gas Riemann problems initial conditions.

\begin{tabular}{lrrll}
\hline Problem & Quantity & Value & Units & Type \\
\hline Sod & $T_{L}$ & 348.43 & $\mathrm{~K}$ & enforced \\
& $T_{R}$ & 278.74 & $\mathrm{~K}$ & enforced \\
& $u_{L}$ & 0 & $\mathrm{~m} / \mathrm{s}$ & enforced \\
& $u_{R}$ & 0 & $\mathrm{~m} / \mathrm{s}$ & enforced \\
& $\rho_{L}$ & 1 & $\mathrm{~kg} / \mathrm{m}^{3}$ & enforced \\
& $\rho_{R}$ & 0.125 & $\mathrm{~kg} / \mathrm{m}^{3}$ & enforced \\
& $p_{L}$ & 100 & $\mathrm{kPa}$ & computed \\
& $p_{R}$ & 10 & $\mathrm{kPa}$ & computed \\
\hline Doub. Exp. & $T_{L}$ & 627.18 & $\mathrm{~K}$ & enforced \\
& $T_{R}$ & 627.18 & $\mathrm{~K}$ & enforced \\
& $u_{L}$ & -316.22 & $\mathrm{~m} / \mathrm{s}$ & enforced \\
& $u_{R}$ & 316.22 & $\mathrm{~m} / \mathrm{s}$ & enforced \\
& $\rho_{L}$ & 1 & $\mathrm{~kg} / \mathrm{m}^{3}$ & enforced \\
& $\rho_{R}$ & 1 & $\mathrm{~kg} / \mathrm{m}^{3}$ & enforced \\
& $p_{L}$ & 180 & $\mathrm{kPa}$ & computed \\
& $p_{R}$ & 180 & $\mathrm{kPa}$ & computed \\
\hline
\end{tabular}

The results are shown in Figures 6 and 7. By looking at the pictures, the exact solution appears to be well captured. As expected, artificial diffusion appears clearly at the contact discontinuity of the Sod problem and at the expansion front ends in both the Sod and double expansion problems. As expected, a minor numerical issue appears at $x=0$ of the double expansion problem in the density trend. It is motivated by the fact that one eigenvalue is zero there, i.e., the velocity $u$. 

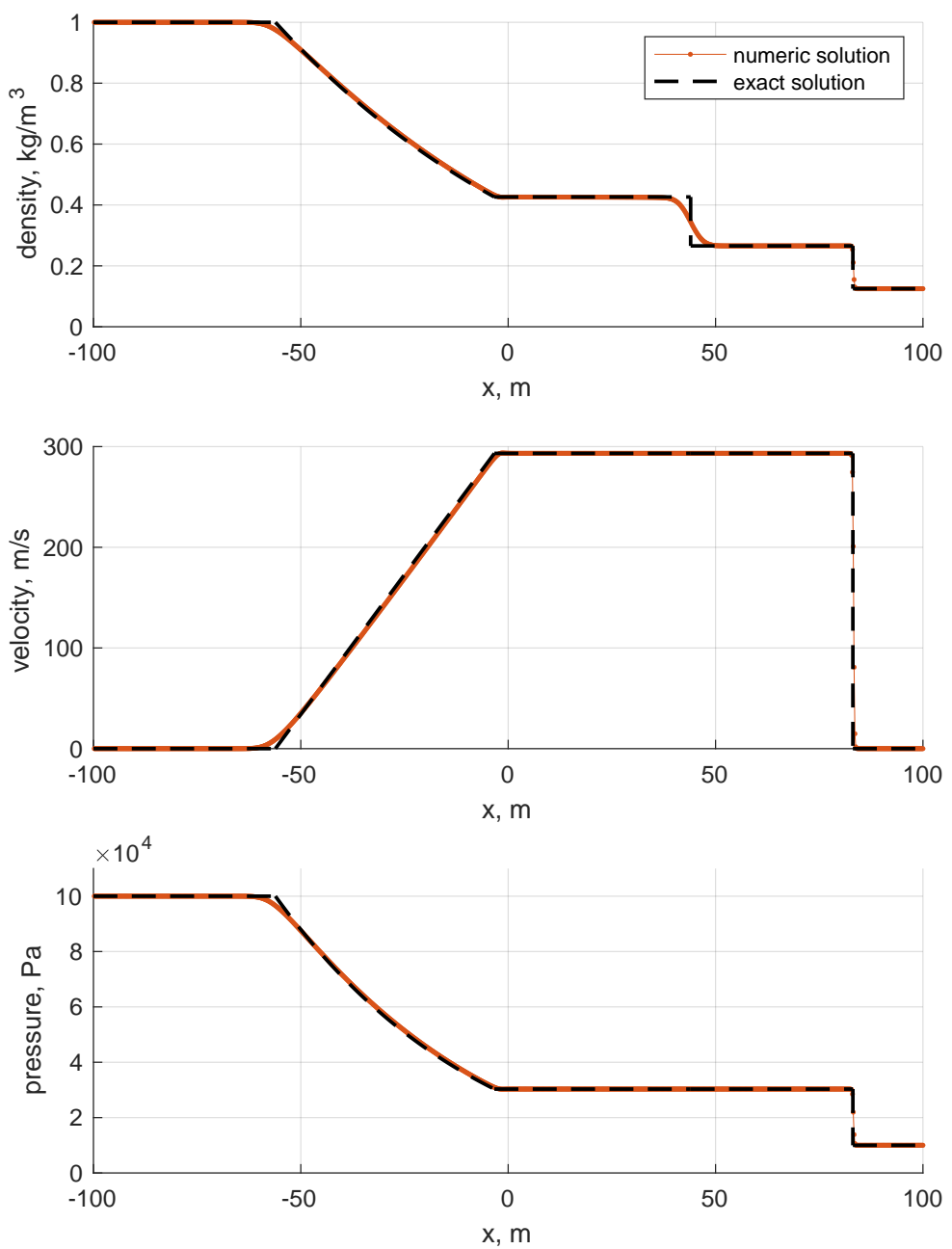

Figure 6. Sod problem. Single species: air. Ideal EoS. Initial conditions listed in Table 3. First order Godunov. One thousand cells.

\subsubsection{Real Gas Riemann Problems}

The next step toward validation is solving a Riemann problem in the real gas regime. Unfortunately, few Riemann problems solutions for real gases are available in the literature. For such a reason, an option to be sought is code-to-code validation, as done, for instance, by Muto et al. [9]. They validated their solutions against that of Arina [27] results. It has to be pointed out that Arina's solution employs a slightly different EoS, since he uses the plain RK formulation, while here, the EoS is set to use the SRK formulation. The problem is a transonic Sod problem, performed in a real gas regime. Initial conditions are shown in Table 4.

Results are shown in Figure 8. They agree to a significant extent with Arina's solution. The slight difference in the EoS is visible in the intermediate states, between the shock and the expansion waves (abscissas $x=[6.5,8.5] \mathrm{m}$ ), and thus, on the contact discontinuity. Nevertheless, both the waves are well captured (see Figure 8). Note that the real and ideal initial conditions in density and temperature correctly result in different pressures (see Figure $8 b)$. 

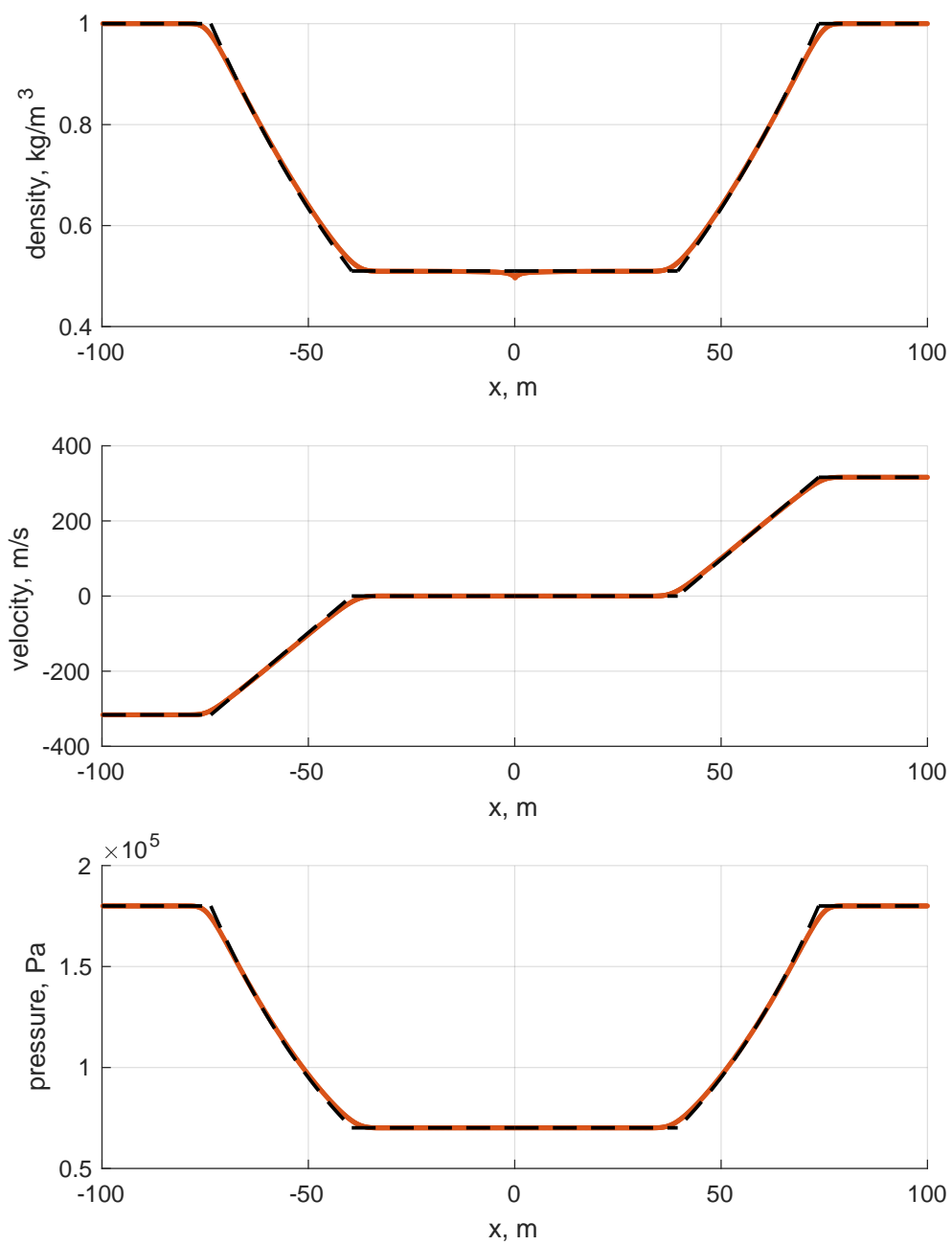

Figure 7. Double symmetric expansion. Single species: air. Ideal EoS. Initial conditions listed in Table 3. First order Godunov. One thousand cells.

Table 4. Real gas transonic Sod problem initial conditions.

\begin{tabular}{crll}
\hline Quantity & Value & Units & Type \\
\hline$T_{L}$ & 892.67 & $\mathrm{~K}$ & enforced \\
$T_{R}$ & 1116.89 & $\mathrm{~K}$ & enforced \\
$u_{L}$ & 0 & $\mathrm{~m} / \mathrm{s}$ & enforced \\
$u_{R}$ & 0 & $\mathrm{~m} / \mathrm{s}$ & enforced \\
$\rho_{L}$ & 348.8 & $\mathrm{~kg} / \mathrm{m}^{3}$ & enforced \\
$\rho_{R}$ & 3.488 & $\mathrm{~kg} / \mathrm{m}^{3}$ & enforced \\
$p_{L}$ & 73760 & $\mathrm{kPa}$ & computed \\
$p_{R}$ & 737.6 & $\mathrm{kPa}$ & computed \\
\hline
\end{tabular}




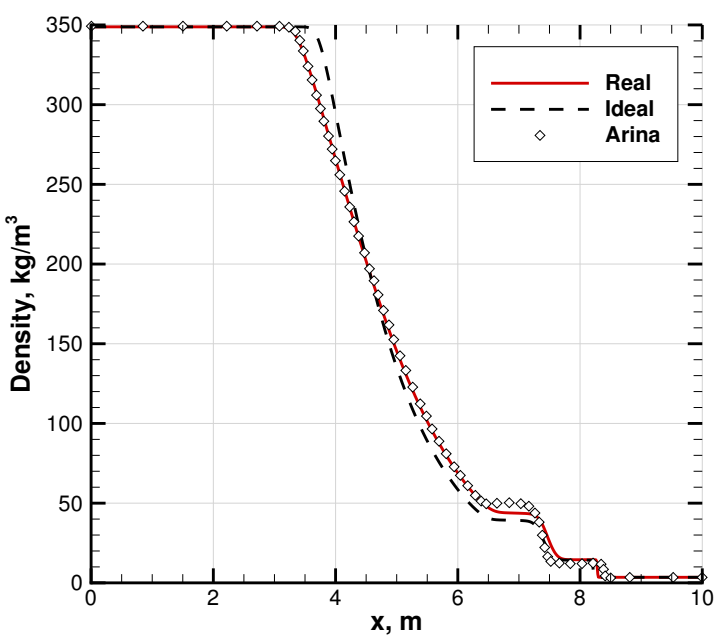

(a) Density.

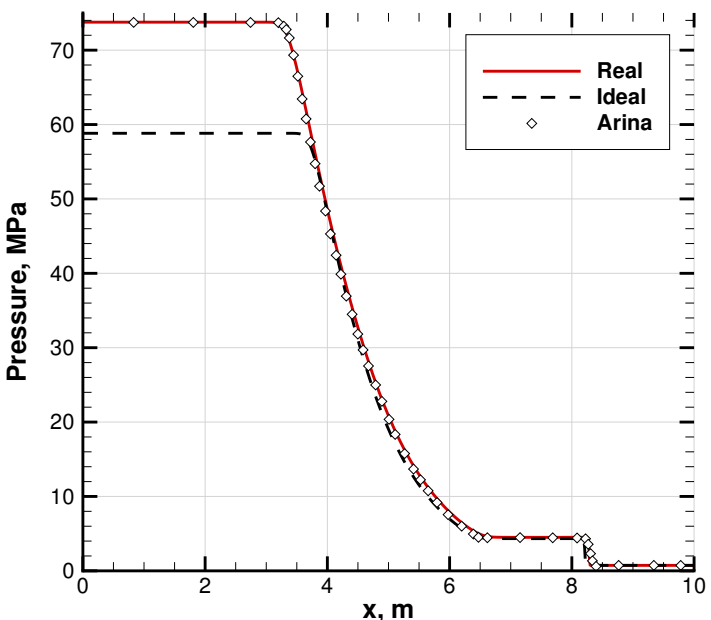

(b) Pressure.

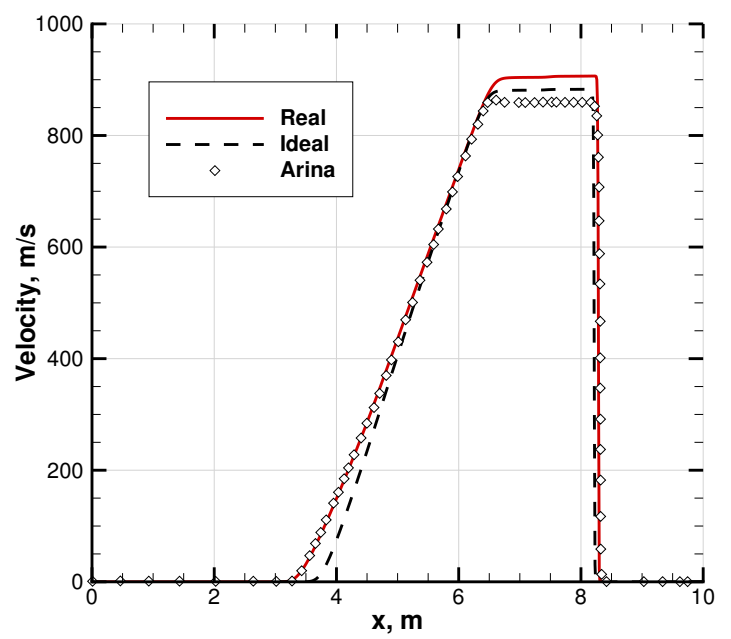

(c) Velocity.

Figure 8. Code-to-code comparison of a transonic Sod problem solution. Literature data are adapted from Arina [27]. EoS is in its SRK mode, while Arina uses plain Redlich-Kwong EoS. Species is pure $\mathrm{CO}_{2}$. Initial conditions are listed in Table 4.

\subsubsection{Acoustic Propagation}

In order to complete the validation, a test involving mixtures is needed. Unfortunately there are no suitable wave propagation validation cases available in the literature that are close to the present problem, to the knowledge of the authors. For such a reason, a simple propagation test is conceived and simulated, that is, a plain linear disturbance propagation in a quiescent closed-closed tube. A disturbance is enforced on the left boundary, on velocity. The amplitude is small enough to keep the flow field in the linear regime, i.e., small perturbations regime. Its amplitude is selected to be $500 \mu \mathrm{m} / \mathrm{s}$; thus, the velocities of propagation can be approximated to the plain speed of sound. Three tests are carried out employing a real $\mathrm{O}_{2}$-ideal $\mathrm{CH}_{4}$ mixture, at $\mathrm{O} / \mathrm{F}=3.4$, at different thermodynamic states representative of actual conditions in the mixing zone of a real engine. Specifically, the pressure is fixed at $12 \mathrm{MPa}$, while the temperature is selected to be 300,600 and $1000 \mathrm{~K}$. The speed of sound is estimated as the space traveled by the disturbance divided by the time interval starting from the moment in which its peak enters in the domain, at the left boundary, and ending at the moment in which it is reflected, at the same boundary, after 
having covered the whole duct back and forth. Such a measure is then compared with refprop values of speed of sound and also with the result provided by the plain EoS in order to assess the error introduced by the RS on such quantity and on propagation dynamics, in general.

Results are shown in Figure 9 for the case with $T=300 \mathrm{~K}$ and listed in Table 5 for all the temperature values. Plots are showed only for the case with $T=300 \mathrm{~K}$ since those at $T=600 \mathrm{~K}$ and $1000 \mathrm{~K}$ only differ from it for the time labels. They show how the speed of sound computed from the results of the propagation test is substantially overlapped onto the value given by the EoS. This means that the RS does not introduce a relevant numerical error which is capable of affecting characteristic times of wave propagation. On the other hand, as expected, propagation tests and plain EoS results differ from refprop to the same extent, due to the error introduced by the cubic formulation. However, the level of agreement can be considered good for those thermodynamic states.

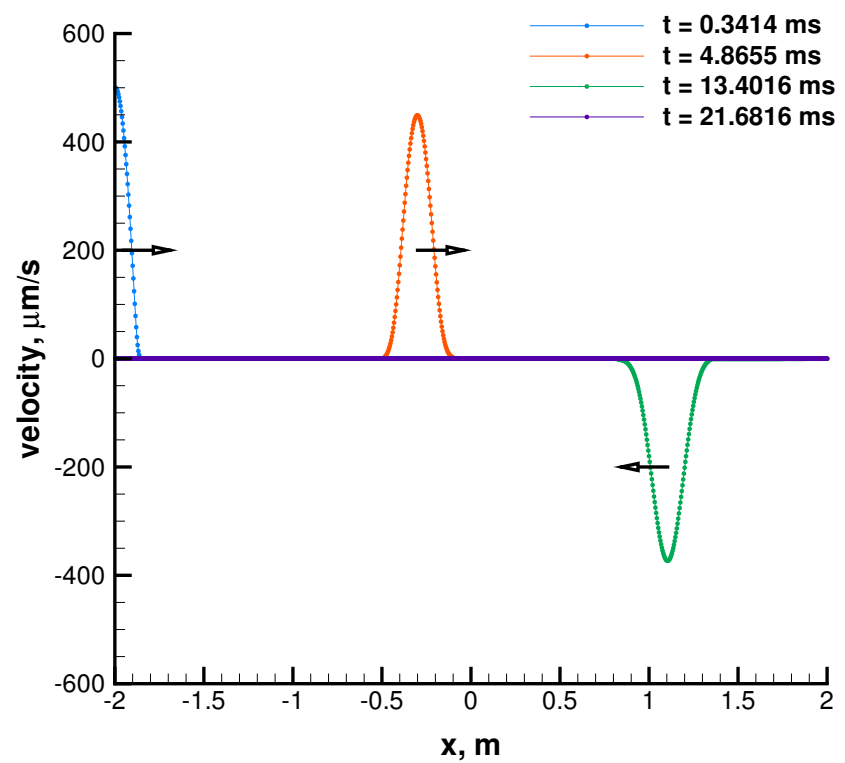

Figure 9. Simple wave propagation problem in a quiescent tube. Velocity is small enough to assume linear behavior of the flow field. The speed of sound is deduced by numerically measuring how long it takes for the signal to propagate back and forth in the tube. Mixture is $\mathrm{O}_{2}-\mathrm{CH}_{4}$ with $\mathrm{O} / \mathrm{F}=3.4$, pressure is $12 \mathrm{MPa}$, while temperature is $300 \mathrm{~K}$.

Table 5. Duct thermodynamic state during acoustic propagation tests. Mixture is made of $\mathrm{O}_{2}-\mathrm{CH}_{4}$ with $\mathrm{O} / \mathrm{F}=3.4$.

\begin{tabular}{crrrll}
\hline Domain State & & Values & & Units & Type \\
\hline Temperature & 300 & 600 & 1000 & $\mathrm{~K}$ & enforced \\
Velocity & 0 & 0 & 0 & $\mathrm{~m} / \mathrm{s}$ & enforced \\
Density & 124.263 & 60.975 & 36.862 & $\mathrm{~kg} / \mathrm{m}^{3}$ & enforced \\
Pressure & 12 & 12 & 12 & $\mathrm{MPa}$ & computed \\
\hline Speed of sound & 374.8793 & 507.3510 & 631.9814 & $\mathrm{~m} / \mathrm{s}$ & numerical propagation \\
Speed of sound & 374.8794 & 507.3490 & 631.9807 & $\mathrm{~m} / \mathrm{s}$ & present EoS value \\
Speed of sound & 373.4200 & 516.6100 & 643.7000 & $\mathrm{~m} / \mathrm{s}$ & (refprop) \\
\hline
\end{tabular}

\section{Discussion and Conclusions}

In this work, a novel thermodynamic modeling approach is derived for dealing with wave propagation in liquid rocket thrust chambers. Such a class of problems is of particular 
interest for the study of high-frequency combustion instability problems. The adopted approach is selected to be low-order modeling-oriented.

Such modeling consists of the adoption of a cubic equation of state for the mixture, which is then significantly simplified to be a special EoS, describing hybrid real/ideal gas mixtures. The reason for this lies in the fact that in dealing with such a class of problems, the only species which is actually needed to be modeled as a real fluid is the oxidizer. Indeed, considering present cryogenic liquid rocket engines, the oxidizer flows into the injectors and then into the chamber at a significantly low temperature, as it comes directly from either the pump assembly or from its tank. On the other hand, the fuel typically reaches the injectors after being used in a regenerative cooling system. For the sake of low order modeling and since it is hotter, an ideal gas modeling can be retained for it. The same applies for hot combustion products.

EoS is validated against refprop data in specific ranges of investigations. Ranges of investigation are those thermodynamic states at which a full-scale liquid rocket engine may operate. Pressure values are chosen to be 6,12 and $18 \mathrm{MPa}$. No combustion regimes are considered in this work since their ideal gas behavior was already verified in previous works. The focus is on the upstream regions of the combustor, namely, injectors posts and recess mixing regions. For such a reason, the temperature is considered in the range $50-400 \mathrm{~K}$ for the pure oxygen into the injector elements, while in the range 300-1000 K, concerning the mixing regions. The results show quite good agreement with refprop in each range of operation, the greatest error recorded being about $20-25 \%$ on the speed of sound, at nearly $100 \mathrm{~K}$.

To solve wave propagation problems, a Riemann solver is developed and adopted. It is cast for the full system of equations, and it is expressed for the primitive variables density and temperature. An important aspect is that its derivation is independent of any EoS, so it can be used for either ideal or real laws.

Concerning the derivation of the RS, it has to be pointed out that the system of equations yielding to its formulation is not uniquely defined. In this framework, the modeling technique which requires the lowest computational cost is preferred. However, different approximations might introduce different numerical errors. Nevertheless, the investigation of such an aspect goes beyond the purposes of this paper and might be interesting for studying in the future.

RS is validated against simple ideal gas Riemann problems in the first place, for two main reasons: the former is that the ideal EoS was already validated in previous works, and the latter is that the exact solution of ideal gas Riemann problems is easy to compute. The results show significant agreement with the exact solution.

After that, EoS and RS are validated together in two ways. The first one is the solution of a real gas Riemann problem. The test case employs carbon dioxide as a fluid, which is something actually far from the present field of application, but a lack of validation test cases in the literature that are closer to our problem has to be reported. The solution is compared with another numerical solution available in the literature, in a code-to-code validation approach. The results show good agreement. The EoS formulation of the solution used for the comparison is slightly different from the one used here, so a slight difference in the computed average states of the Riemann problem is visible and expected. Nevertheless, the acoustic wave system is correctly captured. The second way to synergically validate EoS and RS is performing simple numerical "experiments" of wave propagation in order to measure the speed of sound into the domain. Such numerical measurements can be then compared with refprop values and also with those given by the bare EoS in order to assess the potential numerical errors introduced by the RS and by the governing equations in general. The results are substantially overlapped onto the plain EoS results and, as a consequence, they differ from the refprop dataset to the same extent. 
Author Contributions: Conceptualization, S.D., M.P. and F.N.; methodology, S.D. and M.P.; software, S.D.; validation, S.D.; writing-original draft preparation, S.D.; writing-review and editing, S.D., M.P and F.N.; supervision, F.N.; project administration, F.N. All authors have read and agreed to the published version of the manuscript.

Funding: This research was jointly funded by Sapienza University and the Italian Space AgencyAgenzia Spaziale Italiana (ASI) as part of the research N.2019-4-HH.0 carried out under the framework agreement N.2015-1-Q.0.

Institutional Review Board Statement: Not applicable.

Informed Consent Statement: Not applicable.

Data Availability Statement: The data presented in this study are available on request from the corresponding author.

Acknowledgments: The authors would like to acknowledge the help of the post-graduate research fellow Paolo Maria Zolla for helping in performing the validation procedures.

Conflicts of Interest: The authors declare no conflict of interest.

\section{References}

1. Gröning, S.; Hardi, J.; Suslov, D.; Oschwald, M.; Kim, E. Injector-Driven Combustion Instabilities in a Hydrogen/Oxygen Rocket Combustor. J. Propuls. Power 2016, 32, 560-573. [CrossRef]

2. Harvazinski, M.E.; Huang, C.; Sankaran, V.; Feldman, T.W.; Anderson, W.E.; Merkle, C.L.; Talley, D.G. Coupling between hydrodynamics, acoustics and heat release in a self-excited unstable combustor. Phys. Fluids 2015, 27, 045102. [CrossRef]

3. Wierman, M.; Nugent, N.; Anderson, W.E. Combustion response of a LOX/LCH4 element to tranverse instabilities. In Proceedings of the 47th AIAA/SAE/ASEE Joint Propulsion Conference, San Diego, CA, USA, 31 July-3 August 2011; AIAA Paper 2011-5549. [CrossRef]

4. Amagat, E.H. Sur la détermination de la densité des gaz liquéfiés et de leurs vapeurs saturées-Éléments du point critique de l'acide car bonique. J. Phys. Théor. Appl. 1892, 1, 288-298. [CrossRef]

5. Harvazinski, M.E.; Talley, D.G. Evaluation of Multi-Phase Equations of State for Liquid Rocket Engine Combustion Modeling (Conference Paper with Briefing Charts); Technical Report; Air Force Research Lab, Rocket Lab: Edwards AFB, CA, USA, 2018.

6. Soave, G. Equilibrium constants from a modified Redlich-Kwong equation of state. Chem. Eng. Sci. 1972, 27, 1197-1203. [CrossRef]

7. Robinson, D.B.; Peng, D.Y.; Chung, S.Y. The development of the Peng-Robinson equation and its application to phase equilibrium in a system containing methanol. Fluid Phase Equilibria 1985, 24, 25-41. [CrossRef]

8. Cismondi, M.; Mollerup, J. Development and application of a three-parameter RK-PR equation of state. Fluid Phase Equilibria 2005, 232, 74-89. [CrossRef]

9. Muto, D.; Tsuboi, N.; Terashima, H. Numerical Study of Real Gas Effects on Shock Tube Problems at Supercritical Conditions. Trans. Jpn. Soc. Aeronaut. Space Sci. Aerosp. Technol. Jpn. 2014, 12, Po_2_39-Po_2_44. [CrossRef]

10. Arabi, S.; Trépanier, J.Y.; Camarero, R. A simple extension of Roe's scheme for real gases. J. Comput. Phys. 2017, 329, 16-28. [CrossRef]

11. Arabi, S.; Trépanier, J.Y.; Camarero, R. A simple extension of Roe's scheme for multi-component real gas flows. J. Comput. Phys. 2019, 388, 178-194. [CrossRef]

12. Buffard, T.; Gallouët, T.; Hérard, J.M. A sequel to a rough Godunov scheme: application to real gases. Comput. Fluids 2000, 29, 813-847. [CrossRef]

13. Saurel, R.; Larini, M.; Loraud, J.C. Exact and approximate Riemann solvers for real gases. J. Comput. Phys. 1994, 112, $126-137$. [CrossRef]

14. Kunz, O.; Klimeck, R.; Wagner, W.; Jaeschke, M. The GERG-2004 Wide-Range Equation of State for Natural Gases and Other Mixtures; Published for GERG; Publishing House of the Association of German Engineers: Düsseldorf, Germany, 2007.

15. Kunz, O.; Wagner, W. The GERG-2008 wide-range equation of state for natural gases and other mixtures: An expansion of GERG-2004. J. Chem. Eng. Data 2012, 57, 3032-3091. [CrossRef]

16. Kim, S.K.; Choi, H.S.; Kim, Y. Thermodynamic modeling based on a generalized cubic equation of state for kerosene/LOx rocket combustion. Combust. Flame 2012, 159, 1351-1365. [CrossRef]

17. Toro, E.F. Riemann Solvers and Numerical Methods for Fluid Dynamics: A Practical Introduction; Springer Science \& Business Media: Berlin/Heidelberg, Germany, 2013.

18. Roe, P.L. Approximate Riemann Solvers, Parameter Vectors, and Difference Schemes. J. Comput. Phys. 1981, 43, 357-372. [CrossRef]

19. Roe, P.L.; Pike, J. Efficient construction and utilisation of approximate Riemann solutions. In Proceedings of the Sixth International Symposium on Computing Methods in Applied Sciences and Engineering, VI, Versailles, France, 12-16 December 1983; pp. 499-518. 
20. Glaister, P. An approximate linearised Riemann solver for the Euler equations for real gases. J. Comput. Phys. 1988, 74, 382-408. [CrossRef]

21. Sandler, S.I. Chemical, Biochemical, and Engineering Thermodynamics; John Wiley \& Sons: Hoboken, NJ, USA, 2017.

22. D'Alessandro, S.; Zolla, P.M.; Pizzarelli, M.; Favini, B.; Nasuti, F. A Hybrid Real/Ideal Gas Mixture Model in the Framework of Low Order Modeling of Combustion Instability. In AIAA Propulsion and Energy 2021 Forum; AIAA: Reston, VA, USA, 2021; p. 3623.

23. Lemmon, E.W.; Bell, I.H.; Huber, M.L.; McLinden, M.O. NIST Standard Reference Database 23: Reference Fluid Thermodynamic and Transport Properties-REFPROP; Version 10.0; National Institute of Standards and Technology: Gaithersburg, MD, USA, 2018; [CrossRef]

24. Frezzotti, M.L.; D'Alessandro, S.; Favini, B.; Nasuti, F. Numerical issues in modeling combustion instability by quasi-1D Euler equations. Int. J. Spray Combust. Dyn. 2017, 9, 349-366. [CrossRef]

25. Frezzotti, M.L.; D'Alessandro, S.; Favini, B.; Nasuti, F. Driving mechanisms in low order modeling of longitudinal combustion instability. In American Institute of Aeronautics and Astronautics; AIAA Propulsion and Energy Forum: Cincinnati, OH, USA, 2018; [CrossRef]

26. D'Alessandro, S.; Frezzotti, M.L.; Favini, B.; Nasuti, F. A Multi-dimensional Approach for Low Order Modeling of Combustion Instability in a Rocket Combustor. In Proceedings of the American Institute of Aeronautics and Astronautics, Propulsion and Energy Forum, Cincinnati, OH, USA, 9-11 July 2018; [CrossRef]

27. Arina, R. Numerical simulation of near-critical fluids. Appl. Numer. Math. 2004, 51, 409-426. [CrossRef] 